\title{
Une solution par potentiel de sources pour l'équation des houles à courtes crêtes
}

\author{
PAR R.C. MACCAMY \\ UNIVERSITÉ DE CALIFORNIE, LABORATOIRE D'ÉTUDE DES HOULES
}

English text, p. 379

\begin{abstract}
Le présent mémoire décrit une fonction de Green concernant le problème de la valeur limite tel qu'il se présente dans les questions de diffraction d'ondes à courte crête contournant des obstacles de section transversale limitée. Le problème de diffraction y est formulé de façon précise impliquant une solution unique. La fonction de Green est établie de façon à permettre la représentation du potentiel de vitesses en des points intérieurs du fluide au moyen des valeurs qu'il prend sur l'obstacle; le problème de la diffraction est donc réduit à la solution d'une équation inté-
\end{abstract}

\begin{abstract}
grale de Fredholn du second ordre. A l'aide de cette fonction, l'auteur étudie deux problèmes intéressants de la théorie des ondes de surface, d̀ savoir la génération d'ondes au moyen d'une cloison mobile et leur réflexion par une bande horizontale. Pour le premier de ces problèmes, il obtient des valeurs numériques; pour le second, il donne des indications sur la marche à suivre dans les solutions numériques. En particulier, il formule le problème de la bande de manière à permettre l'application des methodes variationnelles de Schwinger.
\end{abstract}

\section{I. - INTRODUCTION}

Dans la théorie des ondes superficielles, un problème difficile, mais d'importance capitale, est celui de la diffraction autour d'obstacles fixes. Il se présente dans la théorie des bassins, des digues, des jetées, et, accessoirement, dans l'étude des mouvements de navire. Fritz JoHN a abordé le problème dans le mouvement à trois dimensions et réussi à obtenir une équation intégrale comportant des noyaux à singularités. On n'a pas avancé beaucoup depuis, surtout en ce qui concerne les obstacles de dimensions finies.

L'objet de ce rapport est de présenter une solution à sources pour le cas des ondes dites « à courte crête », c'est-à-dire d'ondes douées de périodicité en direction perpendiculaire à celle dans laquelle elles se propagent. Cette solution constitue l'instrument de base quand on s'attaque aux problèmes de diffraction complexe en utilisant les procédés de Fritz JoHN. Sous sa signification physique, elle fournit le potentiel des vitesses d'une ligne de sources dont l'intensité varie périodiquement. Au point de vue mathématique, elle permet d'exprimer le potentiel de l'intérieur du fluide en fonction des données sur la surface de l'obstacle.

C'est au chapitre II que nous présentons la fonction et étudions ses proprićtés, en indiquant aussi les problèmes généraux de la diffraction auxquels elle s'applique. Aux deux chapitres suivants, nous montrons comment appliquer la solution à ces deux problèmes : détermination de la houle 
engendrée par un générateur et coefficient de réflexion d'une barrière horizontale rigide en surface libre. A propos du premier, nous citons quelques valeurs numériques.

On sait que la solution à sources, ou fonction de Green, s'applique à la résolution d'autres problèmes concernant les conditions aux limites. Non seulement elle conduit à des théorèmes d'existence prouvant que le problème est bien formulé, mais elle peut aussi servir à obtenir des solutions approchées. C'est ainsi qu'au chapitre IV, nous montrons que cette méthode permet d'utiliser les calculs de variation de Schwinger.

Nous avons cherché, dans ce rapport, à établir des conceptions ouvertes au calcul numérique. Nous préparons d'ailleurs, dans cet ordre d'idées, un ouvrage à publier ultérieurement.

\section{II. - LA FONCTION DE GREEN}

Avant d'introduire la solution à sources de façon naturelle, nous allons donner un rapide aperçu du problème général de la diffraction des ondes à crête courte en présence d'obstacles cylindriques.

Supposons qu'un fluide incompressible non visqueux remplisse la région considérée :

$$
-\infty \leqslant x \leqslant+\infty \quad-\infty \leqslant Z \leqslant+\infty, \quad 0<y<a,
$$

le plan $y=0$ constituant un fond rigide et $y=a$ une surface libre. Nous supposons, en outre, que le mouvement a été amorcé par un système d'ondes à crêtes courtes, de faible amplitude avançant en direction des $x$ positifs. Pareil système peut être représenté par son potentiel des vitesses :

$$
\Phi^{(i)}=\mathrm{A} \cosh \gamma_{0} y e^{i\left(w_{0} x-\sigma t\right)} \frac{\cos }{\sin } k Z=\operatorname{Re}\left\{\varphi^{(i)} e^{-i \sigma t}\right\} \begin{aligned}
& \cos \\
& \sin
\end{aligned} k Z
$$

A étant une constante et $\gamma_{o} w_{o}$ définis par :

$$
\mathrm{K}=\frac{\sigma^{2}}{g}=\gamma_{o} \tanh \gamma_{o} \quad a, \quad w_{o}^{2}=\gamma_{o}^{2}-k^{2}
$$

On notera que l'équation $(2,2)$ impose à $k$ une limite supérieure, de façon que les ondes progressent en direction des $x$, c'est-à-dire $k<\mathrm{K}$; c'est ce que nous allons supposer dans ce qui suit.

Supposons maintenant qu'à l'intérieur du fluide soit fixé un obstacle cylindrique de section transversale limitée. Le mouvement résultant, le régime permanent ayant été atteint, sera toujours périodique dans le temps, de fréquence $\sigma$. En outre, nous allons réduire le problème aux deux dimen sions en supposant $Z$ lié par des relations de la forme $\cos k Z$ ou $\sin k Z$. Nous pourrons alors exprimer le potentiel des vitesses dans le mouvement résultant par :

$$
\Phi(x, y, Z, t)=\operatorname{Re}\left(\varphi(x, y) e^{-i \sigma t}\right) \begin{aligned}
& \cos \\
& \sin
\end{aligned} k
$$

Il en résulte pour $\varphi$ la relation :

à l'intérieur du fluide.

$$
\varphi_{x x}+\varphi_{y y}-k^{2} \varphi=0
$$

Désignant par $\mathrm{C}_{o}$ la trace de l'obstacle sur le plan $x y$ et par $\mathrm{C}_{\mathrm{F}}$ la partie de $y=a$ qui est extérieure à $\mathrm{C}_{\vartheta}$, nous constatons que $\varphi$ est également soumis aux conditions aux limites suivantes :

$$
\begin{array}{lll}
\varphi_{y}-\mathrm{K}_{\varphi}=0 & \text { sur } & \mathrm{C}_{\mathrm{F}} \\
\varphi_{y}=0 & \text { sur } & y=0 \\
\varphi_{n}=0 & \text { sur } & \mathrm{C}_{o}
\end{array}
$$

$n$ désignant la normale à $\mathrm{C}_{o}$. 
Pour trouver une solution unique, il faudra définir les conditions à l'infini. On peut préyoir que le mouvement sera composé : d'une onde incidente plus une onde réfléchie et une onde transmise; ce qui correspond à une condition de la forme :

$$
\varphi-\varphi^{(i)} \rightarrow \mathrm{T} e^{i w_{0} x} \text { quand } x \rightarrow+\infty, \quad-\varphi^{(i)} \rightarrow \operatorname{Re}^{-i w_{0} x} \text { quand } x \rightarrow-\infty \text {. }
$$

On constate qu'il suffit de satisfaire à la condition la moins rigoureuse.

$$
\lim _{\mathrm{L} \rightarrow \infty}\left\{\int_{0}^{a}\left[\psi_{x}(\mathrm{~L}, y)-i \mathrm{C} \psi(\mathrm{L}, y)\right]^{2} d y+\int_{0}^{a}\left[\psi_{x}(-\mathrm{L}, y)-i \mathrm{C} \psi(-\mathrm{L}, y)\right]^{2} d y\right\}=0
$$

pour un certain $\mathrm{C}>0$, la différence $\psi=\varphi-\varphi^{(i)}$ représentant l'onde dispersée. On peut montrer qu'il n'y a qu'un seul $\varphi$ satisfaisant $(2,4)(2,5)(2,6)(2,7)$ et $(2,9)$ et que cette solution satisfait à $(2,8)$.

La fonction $\mathrm{G}$ de Green qui dépend d'un point-paramètre $\left(x^{\prime}, y^{\prime}\right)$ devra exprimer les valeurs prises par les solutions en ce point au moyen des données caractérisant la surface $\mathrm{C}_{0}$ de l'obstacle. Elle devra satisfaire à $(2,5)$ sur l'ensemble du plan $y=a$, de même qu'à $(2,6)$ pour qu'on n'ait pas à se rapporter aux valeurs que prend la solution sur $y=0$ et $\mathrm{C}_{\mathrm{F}}$. De plus, elle devra présenter une singularité d'un type déterminé pour $(x, y)=\left(x^{\prime} y^{\prime}\right)$ si l'on veut que l'opération :

$$
\int_{-\infty}^{+\infty} \int_{0}^{a}\left(\mathrm{G}_{x x}+\mathrm{G}_{y y}-k^{2} \mathrm{G}\right) \varphi(x, y) d y d x
$$

reproduise les solutions de $(2,4)$. Enfin G devra exprimer la solution aux $|x|$ élevés, donc satisfaire à $(2,9)$.

On trouve la singularité principale de l'équation $(2,4)$ en séparant les variables. Elle est donnée par $\mathrm{K}_{o} \cdot\left(\boldsymbol{k} \sqrt{x^{2}+y^{2}}\right)$ où $\mathrm{K}_{o}$ représente en fait la fonction de Hankel d'ordre 0 et du premier type avec un argument imaginaire pur. Pour ce que nous recherchons, les propriétés importantes pour $\mathbf{K}_{o}(\boldsymbol{Z})$ sont caractérisées par:

$$
\begin{gathered}
\mathrm{K}_{o}(\boldsymbol{Z})=0\left(e^{-z}\right) \text { pour les grands } \mathbf{Z} \\
\mathrm{K}_{o}(\boldsymbol{Z})=\mathrm{A}(\boldsymbol{Z}) \log 1 / \boldsymbol{Z}+\mathbf{B}(\boldsymbol{Z})
\end{gathered}
$$

$\mathrm{A}(z)$ et $\mathrm{B}(z)$ étant des fonctions régulières pour $Z$ réel, et $\mathrm{A}$ telle que $\mathrm{A}(0)=1$.

Il est possible de montrer que la fonction de Green définie comme ci-dessus est unique. Nous allons indiquer une fonction de ce genre. Considérons :

$$
\begin{aligned}
\mathrm{G}\left(x, y, x^{\prime}, y^{\prime}\right)= & \frac{1}{\pi} \int_{\mathrm{C}}^{\infty} \frac{\cosh \gamma y^{\prime}\{\mathrm{K} \sinh \gamma(a-y)-\gamma \cosh \gamma(a-y\}}{\gamma\{\mathrm{K} \cosh \gamma a-\gamma \sinh \gamma a\}} \cos w\left|x-x^{\prime}\right| d w \text { pour } y \geqslant y^{\prime} \\
& \frac{1}{\pi} \int_{\mathrm{C}}^{\infty} \frac{\cosh \gamma y\left\{\mathrm{~K} \sinh \gamma\left(a-y^{\prime}\right)-\gamma \cos \gamma\left(a-y^{\prime}\right\}\right.}{\{\mathrm{K} \cosh \gamma a-\gamma \sinh \gamma a\}} \cos w\left|x-x^{\prime}\right| d w \text { pour } y \leqslant y^{\prime}
\end{aligned}
$$

où $y^{2}=\left(w^{2}+k^{2}\right.$ et où $\mathrm{C}$ est un contour formé de l'axe des $x$ positifs, sauf sur un petit demi-cercle entourant $w_{0}=\sqrt{\gamma_{0}^{2}-k^{2}}$ (lequel est réel si $k<\mathrm{K}$ ). Cette fonction a été donnée par Herns [2] sous forme de développement partiel en fraction.

A l'aide des identités :

$$
\begin{gathered}
\frac{\cosh \gamma \xi\{\mathrm{K} \sin \gamma(a-\zeta)-\gamma \cosh \gamma(a-\zeta)\}}{\gamma\{\mathrm{K} \cos \gamma a-\gamma \sinh \gamma a\}}=\frac{-(\mathrm{K}+\gamma) \cosh \gamma \xi \cosh \gamma \zeta e^{-\gamma a}}{\gamma\{\mathrm{K} \cos \gamma a-\gamma \sinh \gamma a\}} \\
\quad+\frac{e^{-\gamma(\xi-\xi)}}{2 \gamma}+\frac{e^{-\gamma(\xi+\xi)}}{2 \gamma} \\
\int_{0}^{\infty} \frac{e^{-\sqrt{w^{2}+k^{2}} b}}{\sqrt{w^{2}+k^{2}}} \cos w x d w=\mathrm{K}_{o}\left(k \sqrt{\left.x^{2}+b^{2}\right)}\right.
\end{gathered}
$$


l'expression $(2,12)$ peut être transformée en :

$$
\begin{gathered}
\mathrm{G}\left(x, y, x^{\prime}, y^{\prime}\right)=\frac{1}{2 \pi} \mathrm{K}_{0}\left(k \sqrt{\left(x-x^{\prime}\right)^{2}+\left(y+y^{\prime}\right)^{2}}\right)+\frac{1}{2 \pi} \mathrm{K}_{0}\left(k \sqrt{\left(x-x^{\prime}\right)^{2}+\left(y+y^{\prime}\right)^{2}}\right) \\
-\frac{1}{\pi} \int_{0}^{\infty} \frac{(\mathrm{K}+\gamma) \cosh \gamma y \cosh \gamma y^{\prime} e^{-\gamma a}}{\gamma\{\mathrm{K} \cosh \gamma a-\gamma \sinh \gamma a\}} \cos w\left|x-x^{\prime}\right| d w
\end{gathered}
$$

Nous allons voir si c'est vrai. $\mathrm{G}$ possède les propriétés voulues. De $(2,14)$ on voit que $\mathrm{G}_{y}=0$ sur $\gamma=0$; de $(2,13)$ que $\mathrm{G}_{y}-\mathrm{K} \mathrm{G}=0$ sur $y=a$. De plus, $\mathrm{G}$ satisfait à $(2,4)$ sauf au point $\left(x^{\prime} y^{\prime}\right)$ où elle a une singularité du type voulu. Pour voir comment se comporte un $|x|$ grand, il y a lieu de transformer encore $(2,14)$. Nous notons que :

$$
\begin{aligned}
& \text { Res } \\
& w=w_{0}
\end{aligned}\left\{-\frac{1}{\pi} \frac{(\mathrm{K}+\gamma) \cosh \gamma y \cosh \gamma y^{\prime} e^{-\gamma a}}{\gamma(\mathrm{K} \cosh \gamma a-\gamma \sinh \gamma a)}\right\}=-\frac{1}{\pi} \frac{(\mathrm{K}+\gamma) e^{-\gamma_{0} a}}{w_{0} \sinh \gamma_{0} a} \frac{\cosh \gamma_{0} y \cosh \gamma_{0} \underline{y}^{\prime}}{1+\frac{2 \gamma_{o} a}{\sinh 2 \gamma_{0} a}}
$$

et obtenons, en modifiant le contour $\mathrm{C}$ :

$$
\begin{aligned}
& \mathrm{G}\left(x, y, x^{\prime}, y^{\prime}\right)=\frac{1}{2 \pi} \mathrm{K}_{o}\left(k \sqrt{\left(x-x^{\prime}\right)^{2}+\left(y-y^{\prime}\right)^{2}}\right)+\frac{1}{2 \pi} \mathrm{K}_{o}\left(k \sqrt{\left(x-x^{\prime}\right)^{2}+\left(y+y^{\prime}\right)^{2}}\right. \\
& -\frac{i(\mathrm{~K}+\gamma) e^{-\gamma_{o} a}}{w_{o} \sinh \gamma_{o} a} \frac{\cosh \gamma_{0} y \cosh \gamma_{n} \underline{y}^{\prime}}{1+\frac{2 \gamma_{0} a}{\sinh 2 \gamma_{0} a}} e^{i w_{0}\left|x-x^{\prime}\right|}-\frac{1}{\pi} \operatorname{Re} \int_{\mathrm{C}^{\prime}}^{\infty} \frac{(\mathrm{K}+\gamma) \cosh \gamma y \cosh \gamma y^{\prime}}{\gamma(\mathrm{K} \cosh \gamma a-\gamma \sinh \gamma a)} e^{i w_{0}\left|x-x^{\prime}\right| d w}
\end{aligned}
$$

où $\mathrm{C}^{\prime}$ est un contour situé dans le premier ou le quatrième quadrant selon que $x-x^{\prime}$ est $>0$ ou $<0$. D'après $(2,10)$, on a alors :

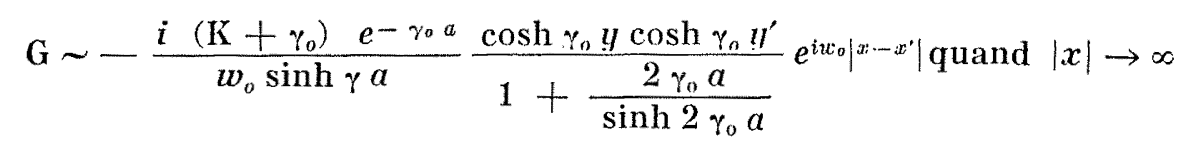

Mais l'équation $(2,2)$ a, en plus des racines réelles $\pm \gamma_{0}$, une infinité de racines imaginaires $\pm i \rho_{n}, n=1,2 \ldots$. Nous aurions donc pu, bien que nous ne l'ayons pas fait, utiliser la transformation ci-dessus pour obtenir le développement du terme intégral en une série de fonctions *.

Dans le cas d'une profondeur infinie, on pourra pousser la transformation un peu plus loin et obtenir une expression de $\mathrm{G}$ ne comprenant que des intégrales réelles. Pour plus de commodité, nous changeons de système de coordonnées de façon à avoir $y$ mesuré en direction verticale vers le bas à partir de la surface libre. L'équation $(2,15)$ devient alors :

$$
\begin{aligned}
\mathrm{G}\left(x, y, x^{\prime}, y^{\prime}\right)=\mathrm{K}_{o}\left(k \sqrt{\left(x-x^{\prime}\right)^{2}+\left(y-y^{\prime}\right)^{2}}\right) & -\frac{2 i \mathrm{~K}}{w_{o}} e^{-\mathrm{K}\left(y+y^{\prime}\right)} e^{i w_{o}}\left|x \cdots x^{\prime}\right| \\
& -\frac{1}{\pi} \operatorname{Re} \int_{\mathrm{C}^{\prime}}^{\infty} \frac{(\gamma+\mathrm{K})}{\gamma(\mathrm{K}-\gamma)} e^{-\gamma\left(y+y^{\prime}\right)} e^{i w\left(x-x^{\prime}\right)} d w,
\end{aligned}
$$

$\mathrm{C}^{\prime}$ correspondant cette fois-ci à l'axe imaginaire positif, sauf sur un petit demi-cercle au demi-plan droit entourant la singularité $\gamma=0$, c'est-à-dire $w=i k$. Le calcul de la partie réelle donne :

$$
\begin{aligned}
& \mathrm{G}\left(x, y, x^{\prime}, y^{\prime}\right)=\frac{1}{2 \pi} \mathrm{K}_{o}\left(k \sqrt{\left(x-x^{\prime}\right)^{2}+\left(y-y^{\prime}\right)^{2}}\right)+\frac{1}{2 \pi} \mathrm{K}_{o}\left(k \sqrt{\left(x-x^{\prime}\right)^{2}+\left(y+y^{\prime}\right)^{2}}\right) \\
& +\frac{2 i \mathrm{~K}}{w_{o}} e^{-\mathrm{K}\left(y+y^{\prime}\right)} e^{i w\left(x-x^{\prime}\right)}-\frac{2 \mathrm{~K}}{\pi} \int_{k}^{\infty}\left[\frac{\gamma \sin \gamma\left(y+y^{\prime}\right)+\mathrm{K} \cos \gamma\left(y+y^{\prime}\right)}{\gamma\left(\gamma^{2}+\mathrm{K}^{2}\right)} e^{-\tau\left(x-x^{\prime}\right)}\right] d \tau
\end{aligned}
$$

oủ $\gamma=\sqrt{\tau^{2}-k^{2}}$ et où les valeurs principales de l'intégrale sont considérées, et où il est fait usage de $(2,13)$.

$\left(^{*}\right) \cos \rho^{n}\left(y-y^{\prime}\right) e^{p_{n}}\left|x-x^{\prime}\right|, \cos \rho_{n}\left(y+y^{\prime}\right) e^{-\rho_{n}}\left|x-x^{\prime}\right|$ 


\section{III. - GENERATION DE HOULE PAR PAROIS MOBILES}

En première approximation nous allons considérer la génération de houle dans un bassin à l'aide d'une paroi verticale. Nous supposons que la paroi est divisée sur la longueur en éléments dont chacun peut être animé d'un mouvement ayant sa phase et son amplitude propres. Si les éléments sont suffisamment petits, on pourra réaliser n'importe quelle forme arbitraire de répartition des vitesses horizontales de la paroi. Nous supposons également que la paroi est au total assez longue pour négliger les effets d'extrémité. Il s'agit de déterminer le mouvement de la paroi, et l'effort nécessaire à la réalisation de ce mouvement pour obtenir une houle donnée.

La solution du problème est donnée par les expressions suivantes, généralisation des recherches de Havelock [3] et de Kennard [4].

Théonème. La fonction $\varphi(x, y)$, définie par

$$
(x, y)=-2 \int_{0}^{\infty} f\left(y^{\prime}\right) \mathrm{G}\left(x, 0, x^{\prime}, y^{\prime}\right) d y^{\prime},
$$

est une solution du problème :
a) $\varphi_{x x}+\varphi_{y y}-k^{2} \varphi=0$
b) $\varphi_{\mu l}=0$
c) $\varphi_{y}-\mathrm{K} \varphi=0$
d) $\varphi \rightarrow \mathrm{A} \cosh \gamma_{\rho} y e^{i w_{0} x}$
e) $\lim _{x \rightarrow 0^{+}} \frac{\partial \varphi}{\partial x}=f(y)$

$$
\begin{array}{cl}
\text { pour } & 0<x<\infty, 0<y<a \\
\text { sur } & y=0, \quad x>0 \\
\text { sur } & y=a, \quad x>0 \\
\text { quand } & x \rightarrow \infty
\end{array}
$$$$
\text { pour } 0<y<a \text { et } f(y) \text { continu dans } 0<y<a \text {. }
$$

Démonstration. Les trois premières propriétés résultent immédiatement de celles de G; la d) résulte de $(2,16)$ avec :

$$
\mathrm{A}=+\frac{2 i(\mathrm{~K}+\gamma)}{w_{0} \sinh \gamma_{0} a} \frac{e^{-\gamma_{0} a}}{1+\frac{2 \gamma_{0} a}{\sinh 2 y_{0} a}} \int_{0}^{a} f\left(y^{\prime}\right) \cosh \gamma_{o} y^{\prime} d y^{\prime}
$$

De $(2,14)$ on déduit :

$$
\lim _{x \rightarrow 0^{+}} \frac{\partial \varphi}{\partial x}=\frac{1}{\pi} \lim _{x \rightarrow 0} \int_{0}^{a} f\left(y^{\prime}\right) \frac{\partial}{\partial x} \mathrm{~K}_{o}\left(k \sqrt{\left(x-x^{\prime}\right)^{2}+\left(y-y^{\prime}\right)^{\overline{2}}}\right) d y^{\prime}
$$

Au moyen de $(2,11)$ et en nous basant sur le caractère continu de $f$, nous pouvons réduire cette expression davantage :

$$
\lim _{x \rightarrow 0^{+}} \frac{\partial \varphi}{\partial x}=\frac{1}{\pi} \lim _{x \rightarrow 0^{+}} f(y) \int_{-\infty}^{+\infty} \frac{x d x}{x^{2}+\left(y-y^{\prime}\right)^{2}}
$$

Nous basant sur le fait que :

nous obtenons :

$$
\int_{-\infty}^{+\infty} \frac{x d x}{x^{2}+\left(y-y^{\prime}\right)^{2}}=\begin{array}{r}
\pi \text { pour } x>0 \\
-\pi \text { pour } x<0
\end{array}
$$

$$
\lim _{x \rightarrow 0^{+}} \frac{\partial \varphi}{\partial x}=f(y)
$$

qui vient compléter la démonstration et comporte ce corollaire précieux :

Corollaire. Si :

$$
\varphi(x, y)=-2 \int_{0}^{a} f\left(y^{\prime}\right) \mathrm{K}_{o}\left(k \sqrt{\left(x-x^{\prime}\right)^{2}+\left(y-y^{\prime}\right)^{2}}\right) d y^{\prime}
$$


et si $f(y)$ est continue, on a :

$$
\lim _{x \rightarrow 0^{+}} \frac{\partial \varphi}{\partial x}=f(y), \quad \quad \lim _{x \rightarrow 0^{+}} \frac{\partial \varphi}{\partial x}=-f(y)
$$

Cette formule $(3,3)$ est un cás particulier d'un théorème plus général que nous n'allons pas exposer ici. Les intégrales du type ci-dessus représentent des «lignes de sources », analogues à celle que l'on rencontre dans la théorie classique du potentiel; la formule $(3,3)$ ne fait qu'exprimer la condition de discontinuité qui se présente au passage de cette ligne.

Nous pouvons maintenant aborder le problème de la génération de la houle. Pour simplifier, nous supposons que le dispositif générateur est du type plongeur, donc que $f(y)=B$ (constante). Nous désignons par $v(y, z, t)$ la vitesse horizontale de la paroi et par $\eta_{\infty}$ la cote de la surface libre à l'infini. Considérant que :

$$
\eta=\left.\frac{1}{g} \frac{\partial \Phi}{\partial t}\right|_{y=0}
$$

nous pouvons, grâce au théorème précédent, envisager plusieurs cas.

$$
\begin{aligned}
& \text { CAS }(1),-\mathrm{B} \text { réel : } \\
& v_{1}{ }^{c}=\mathrm{B} \cos \sigma t \cos k z, \quad \eta_{\infty}{ }^{1 .} \mathrm{c}=\frac{2 \sigma \mathrm{B} / g}{w_{0}\left[1+\left(2 \gamma_{0} a / \sinh \gamma_{0} a\right)\right\rceil} \cos \left(w_{0} x-\sigma t\right) \cos k z \\
& v_{1}{ }^{s}=\mathrm{B} \cos \sigma t \sin k z, \quad \eta_{\infty}{ }^{1, s}=\frac{2 \sigma \mathrm{B} / g}{w_{0}\left\lceil 1+\left(2 \gamma_{0} a / \sinh \gamma_{0} a\right)\right\rceil} \cos \left(w_{o} x-\sigma t\right) \sin k z
\end{aligned}
$$

Cas (2). - B imaginaire :

$$
\begin{aligned}
& v_{2}{ }^{c}=\mathrm{B} \sin \sigma t \cos k z, \quad r_{\infty}{ }^{2} e=\frac{2 \sigma \mathrm{B} / g}{w_{0}\left[1+\left(2 \gamma_{0} a / \sinh \gamma_{0} a\right)\right]} \sin \left(w_{0} x-\sigma t\right) \cos k z \\
& v_{2}{ }^{s}=\mathrm{B} \sin \sigma t \sin k z, \quad \eta_{\infty}{ }^{2, s}=\frac{2 \sigma \mathrm{B} / g}{w_{0}\left[1+\left(2 \gamma_{0} a / \sinh \gamma_{0} a\right)\right\rceil} \sin \left(w_{0} x-\sigma t\right) \sin k z
\end{aligned}
$$

CAS (3). - Superposition de $v_{1}^{c}$ et $v_{2}^{s}$ :

$$
\begin{gathered}
v=v_{1}{ }^{e}+v_{2}{ }^{s}=\mathrm{B} \cos (k z-\sigma t) \\
\eta_{\infty}=\eta_{1 \infty}{ }^{1, c}+\eta^{2, s}=\frac{2 \sigma \mathrm{B} / g}{w_{0}\left[1+\left(2 \gamma_{0} \alpha / \sinh \gamma_{0} \alpha\right)\right]} \cos \left(w_{o} x-k z-\sigma t\right)
\end{gathered}
$$

Les deux premiers cas se présentent quand tous les éléments de la paroi sont en phase, mais avec différentes amplitúdes. La houle résultante constituera, à une distance suffisante de la paroi, un système à crêtes courtes se propageant vers l'extérieur.

Au contraire, dans le troisième cas, les éléments ont des mouvements de même amplitude, déphasés, et donneront lieu cette fois-ci à une houle cylindrique se propageant vers l'extérieur sous un angle $\operatorname{arctg}^{-1} \mathrm{k} / \mathrm{w}$ relativement à l'axe des $x$, à une fréquence $\sigma$ et une longueur d'onde $2 \pi / \gamma_{0}$.

Si $y_{o}$ désigne l'amplitude du mouvement de la paroi, nous déduisons de $(3,5)$ et $(3,6)$ :

$$
\frac{\left|\eta_{\infty}\right|}{\mu_{o}}=\frac{2 \mathrm{~K}}{w_{o}} \frac{1}{1+\left(2 \gamma_{i j} a / \sinh 2 \gamma_{0} a\right)}
$$

pour une amplitude $\left|\eta_{\infty}\right|$ de la houle cylindrique et dans le cas (3). Nous pouvons choisir à volonté $\sigma$ et $\mathrm{K}$; nous pouvons donc prendre comme paramètre $\mathrm{K} a=\left(\sigma^{2} \alpha / 2 \pi g\right)$ et $k a$. Les paramètres importants pour le calcul sont $\mathrm{K} a$, ainsi que $k / w_{o}=\beta$, qui, ainsi que nous l'avons vu, définit la direction de propagation de la houle cylindrique.

Dans une eau de profondeur infinie $\gamma_{o}=\mathrm{K}$; donc $\mathrm{K} a / 2 \pi$ est le rapport de la profondeur à la longueur d'une houle à crêtes longues en eau profonde de fréquence $\sigma$. En pareil cas, nous pouvons entrer dans les tables de WiEgeL [5] avec comme argument $\mathrm{K} a / 2 \pi$, au lieu de $d / \mathrm{L}_{0}$. 
Sur la figure 1 sont représentés quelques-uns des résultats obtenus. La figure 2 correspond au cas d'un batteur à volets articulé au fond. A noter que ce dispositif générateur de houle perd de son efficacité quand on accentue l'angle sous lequel l'onde se propage. En fait, dès que $\theta$ approche de $45^{\circ}$, le rapport $(3,7)$ ci-dessus tend vers zéro. En cherchant à réaliser des angles plus grands, on n'arrive qu'ạ engendrer des ondes dites «marginales», qui s'évanouissent selon une formule exponentielle au loin du générateur. Dans un ouvrage récent, Ursell [6] considère un cas analogue avec un cylindre circulaire immergé.

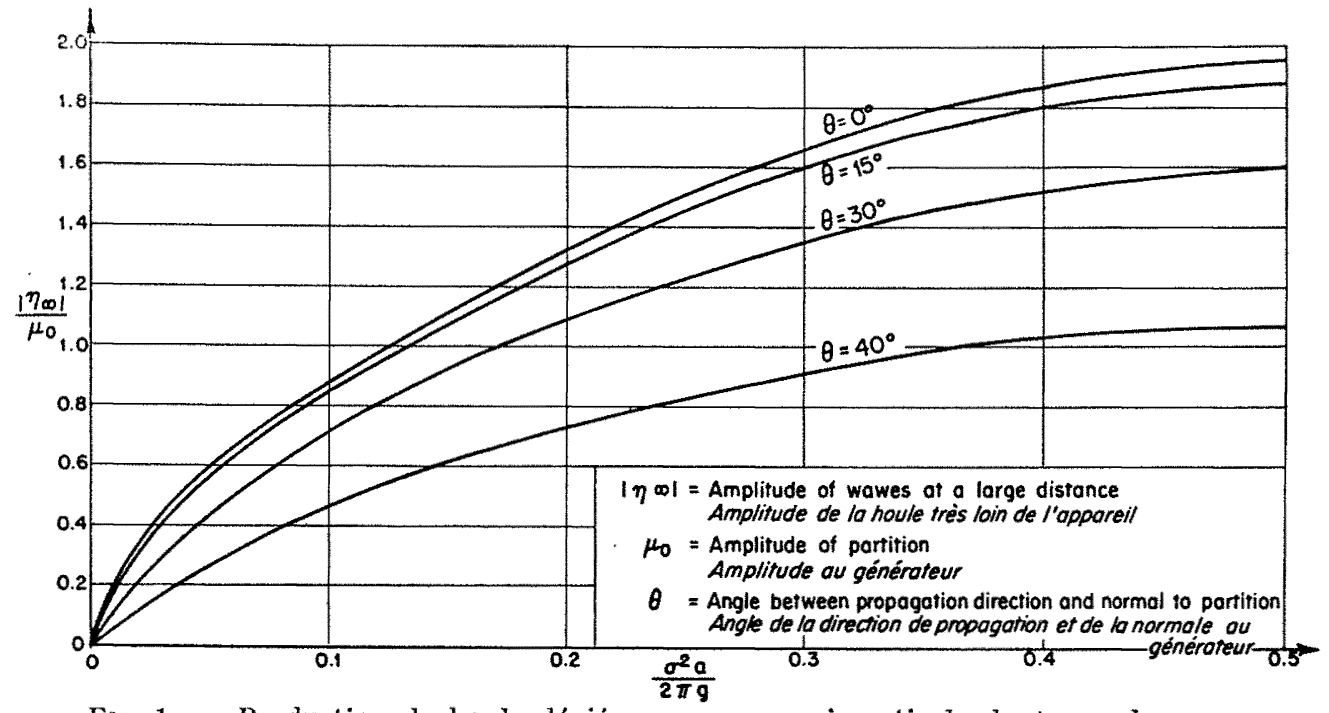

Fir. 1. - Production de houle déviée par une paroi verticale du type plongeur.

Production of Waves at an Angle by a vertical Partition of plunger Type.

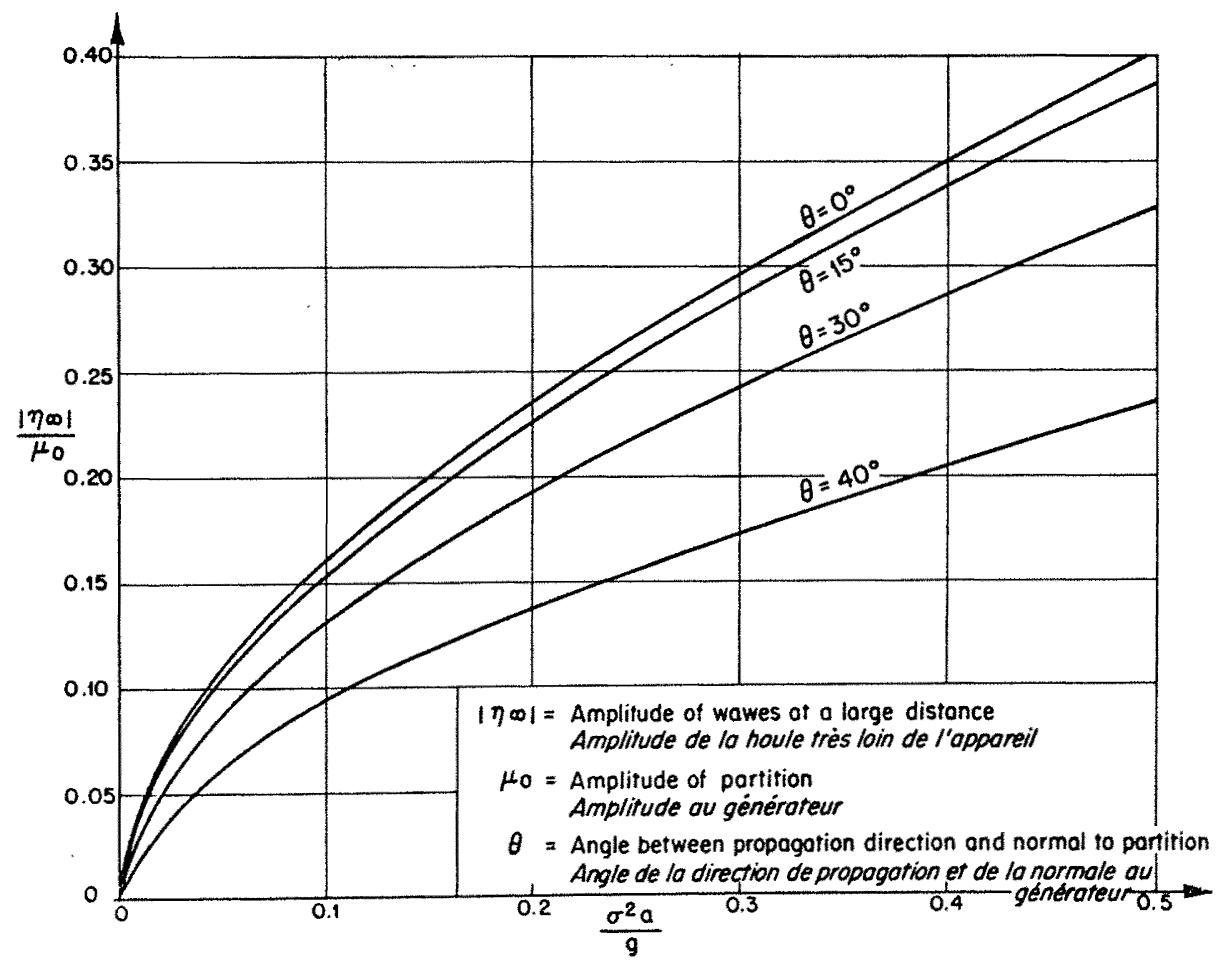

Fig. 2. - Production de houle déviée par une paroi verticale du type batteur.

Production of Waves at an Angle by a vertical Partition of fapper Type.

Pour avoir la pression sur la cloison, nous pouvons utiliser :

$$
p(0, y, z, t)=\left.\rho g \frac{\partial \Phi}{\partial t}\right|_{x=0}
$$


et nous obtenons :

$$
p=-\imath g \sigma \operatorname{Re}\left\{i \mathrm{~B} \int_{0}^{a} \mathrm{G}\left(o, y, o, y^{\prime}\right) d y^{\prime} e^{-i \sigma t}\right\} \begin{aligned}
& \cos \\
& \sin
\end{aligned} z,
$$

intégrale que l'on peut traiter par quadrature.

\section{IV. - REFLEXION PAR UNE BANDE}

Comme nouvelle application, nous allons considérer la réflexion de houles à crêtes courtes à partir d'une bande de profondeur finie, fixée rigidement sur la surface libre. Sous forme mathématique, le problème se présente comme suit :

$$
\begin{aligned}
& \varphi_{x x}+\varphi_{y y}-k^{2} \varphi=0 \\
& \varphi_{y}=0 \\
& \varphi_{y}-\mathrm{K} \varphi=0 \\
& \varphi_{y}=0 \\
& \varphi-\mathbf{A} \cosh \gamma_{o} y e^{i w_{o} x}
\end{aligned}
$$

$$
\begin{aligned}
\text { pour } & 0<y<a \\
\text { sur } & y=0 \\
\text { sur } & y=a, \quad|x|>b \\
\text { sur } & y=a, \quad|x|<b
\end{aligned}
$$

satisfait à une condition de la forme $(2,9)$.

Nous notons maintenant que l'analyse de Fritz JoHn peut être conduite à peu près sans changement et donne un théorème d'existence tout à fait général applicable au problème de diffraction, dont il est question au chapitre II, mais qui exige néanmoins que la surface constituant obstacle soit perpendiculaire à la surface libre au lieu d'intersection, condition qui n'est pas satisfaite dans le présent problème. Notre raisonnement constituera par lui-même un tel théorème d'existence pour le cas considéré.

Considérons la fonction :

$$
\psi\left(x^{\prime}, y^{\prime}\right)=\int_{-b}^{+b} f(x) \mathrm{G}\left(x, a, x^{\prime}, y^{\prime}\right) d x+\mathrm{A} \cosh \gamma_{o} y^{\prime} e^{i w_{\theta} x}
$$

où $f(x)$ est une fonction que nous définirons par la suite. De $(2,16)$ nous déduirons :

$$
\begin{gathered}
\psi\left(x^{\prime}, y^{\prime}\right) \sim \mathrm{A} \cosh \gamma_{o} y^{\prime} e^{i w_{o} x^{\prime}}-i \frac{\left(\mathrm{K}+\gamma_{0}\right) \cdot e^{-\gamma_{o} a}}{w_{o} \sinh \gamma_{o} a} \frac{\cosh \gamma_{o} y^{\prime}}{1+\left(2 \gamma_{o} a / \sinh 2 \gamma_{o} a\right)} e^{i w_{o} x^{\prime}} \int_{-b}^{+b} f(x) e^{-i w_{o} x} d x \\
=\mathrm{A} \cosh \gamma_{o} y^{\prime} e^{i w_{o} x^{\prime}}+\mathrm{T}_{1} \cosh \gamma_{o} y^{\prime} e^{i w_{o} x^{\prime}} \text { quand } x \rightarrow+\infty, \\
\psi\left(x^{\prime}, y^{\prime}\right) \sim \mathrm{A} \cosh \gamma_{o} y^{\prime} e^{i w_{o} x^{\prime}}-\frac{i\left(\mathrm{~K}+\gamma_{0}\right) e^{-\gamma_{o} a}}{w_{o} \sinh \gamma_{o} a} \frac{\cosh \gamma_{o} y^{\prime}}{1+\left(2 \gamma_{o} a / \sinh 2 \gamma_{o} a\right)} e^{-i w_{o} x^{\prime}} \int_{-b}^{+b} f(x) e^{-i w_{o} x} d x \\
=\mathrm{A} \cosh \gamma_{o} y^{\prime} e^{i w_{o} x^{\prime}}+\mathrm{R} \cosh \gamma_{o} y^{\prime} e^{-i w_{o} x^{\prime}} \text { quand } \quad x \rightarrow-\infty .
\end{gathered}
$$

Ainsi donc, $\psi\left(x^{\prime} y^{\prime}\right)$ satisfait à $(4,5), \mathrm{R}$ représentant une houle réfléchie, tandis que $\mathrm{T}_{1} \cosh$ $\gamma_{o} y^{\prime} e^{i w_{o} x^{\prime}}$ se combine à la houle incidente pour engendrer une houle transmise. Par différentiation directe, on vérifie que $\psi$ satisfait également à $(4,1)(4,2)$ et $(4,3)$. Pour que $\psi\left(x^{\prime}, y^{\prime}\right)$ devienne une solution de notre problème, il suffira de montrer que l'on peut choisir $f(x)$ de manière à satisfaire à $(4,4)$.

De l'équation $(2,12)$ définissant $G$, nous déduisons :

$$
\frac{\partial \mathrm{G}}{\partial y^{\prime}}\left(x, a, x^{\prime}, y^{\prime}\right)=-\frac{1}{\pi} \int_{\dot{\sim} \rightarrow}^{\infty} \frac{\sinh \gamma y^{\prime}}{\mathrm{K} \cosh \gamma a-\gamma \sinh \gamma a} \cos w\left|x-x^{\prime}\right| d w .
$$


D'autre part, en différentiant l'identité $(2,13)$ par rapport à $b$, nous voyons que cette expression peut aussi s'écrire :

$$
\begin{aligned}
\frac{\partial \mathrm{G}}{\partial y^{\prime}}\left(x, a, x^{\prime}, y^{\prime}\right)= & +\frac{1}{\pi} \frac{\partial}{\partial y^{\prime}}\left\{\mathrm{K}_{o}\left(k \sqrt{\left(x-x^{\prime}\right)^{2}+\left(a-y^{\prime}\right)^{2}}\right)\right\} \\
& -\frac{1}{\pi} \int_{\sim \rightarrow}^{\infty}\left[\frac{\sinh \gamma y}{\mathrm{~K} \cosh \gamma a-\gamma \sinh \gamma a}+e^{-\gamma\left(a-y^{\prime}\right)}\right] \cos w\left|x-x^{\prime}\right| d w .
\end{aligned}
$$

Nous allons maintenant énoncer sans démonstration la conclusion suivante dont une partie a été démontrée, dans son essence, au chapitre III.

Lemme 1. - La fonction :

$$
\Phi\left(x^{\prime}, y^{\prime}\right)=\int_{-b}^{+b} f(x) \mathrm{K}_{o}\left(k \sqrt{\left(x-x^{\prime}\right)^{2}+\left(a-y^{\prime}\right)^{2}}\right) d x
$$

quand $f(x)$ est continu entre les limites $-b<x<+b$, est une solution de $\Phi_{x^{\prime} \underline{x}^{\prime}}+\Phi_{y^{\prime} y^{\prime}}-k^{2} \Phi=0$ pour $y^{\prime}<0$; elle est continue sur $y^{\prime}=a$, avec $-b<x<+b$, quand elle est définie comme sa valeur limite à partir de $y^{\prime}<a$ et satisfait à :

$$
\lim _{\substack{y^{\prime} \rightarrow a \\ y^{\prime}<a}} \frac{\partial \Phi(x, y)}{\partial y^{\prime}}=\pi f(x) \text { pour }-b<x^{\prime}<b .
$$

Ce lemme, conjointement avec l'équation $(4,10)$, nous permet d'écrire :

$$
\lim _{y^{\prime} \uparrow a} \frac{\partial \psi\left(x^{\prime}, y^{\prime}\right)}{\partial y^{\prime}}=f\left(x^{\prime}\right)+\int_{-b}^{+b} f(x) \mathrm{K}\left(x, x^{\prime}\right) d x+i w_{o} \mathrm{~A} \cosh \gamma_{o} a e^{i n w_{o} x}
$$

où :

$$
\begin{aligned}
& \mathrm{K}\left(x, x^{\prime}\right)=-\frac{1}{\pi} \int_{0}^{\infty}\left[\frac{\gamma \sinh \gamma a}{\mathrm{~K} \cosh \gamma a-\gamma \sinh \gamma a}+1\right] \cos w\left|x-x^{\prime}\right| d w \\
&=-\frac{\mathrm{K}}{\pi} \int_{0}^{\infty} \frac{\cosh \gamma a \cos w\left|x-x^{\prime}\right| d w}{\mathrm{~K} \cosh \gamma a-\gamma \sinh \gamma a}
\end{aligned}
$$

En posant :

$$
\lim _{y^{\prime} \uparrow^{a}} \frac{\partial \psi(x, y)}{\partial y^{\prime}}=0
$$

nous obtenons une équation intégrale pour déterminer $f(x)$.

Examinons la nature du noyau $\mathrm{K}\left(x, x^{\prime}\right)$. Nous écrivons :

$$
\mathrm{K}\left(x, x^{\prime}\right)=-\frac{1}{\pi} \int_{\dot{\mathrm{C}}^{\prime}}^{\infty} \frac{\cos w\left|x-x^{\prime}\right| d w}{1-(\gamma / \mathrm{K})} \frac{1}{\pi \mathrm{K}} \int_{\mathrm{C}^{\prime \prime}}^{\infty} \frac{\gamma \cos w\left(x-x^{\prime}\right)[\tanh \gamma a-1]}{[1-(\gamma / \mathrm{K}) \tanh \gamma a][1-(\gamma / \mathrm{K})]} d w
$$

où $\mathrm{C}^{\prime}, \mathrm{C}^{\prime \prime}$ correspondent à la partie positive de l'axe réel, à l'exception des demi-cercles au voisinage des points singuliers dans le demi-plan inférieur. Toutes les difficultés relatives à $x=x^{\prime}$ sont contenues dans le premier terme. En procédant comme précédemment, nous trouvons :

$$
\mathrm{K}\left(x, x^{\prime}\right)=\frac{1}{\pi} \int_{0}^{\infty} \frac{\cos w\left(x-x^{\prime}\right)}{\sqrt{w^{2}+k^{2}}} d w+\ldots-\ldots
$$

le pointillé indiquant des termes qui restent limités quand $x$ tend vers $x^{\prime}$. On voit donc d'après l'équation $(2,13)$, que $\mathrm{K}\left(x, x^{\prime}\right)$ se comporte comme $\mathrm{K}_{0}\left(k\left|x-x^{\prime}\right|\right)$ au voisinage de $x^{\prime}=x$ et a une singularité logarithmique en cet endroit. 
Nous avons ainsi affaire à l'équation intégrale :

$$
-i w_{o} \mathrm{~A} \cosh \gamma_{o} a e^{i w_{0} x}=f\left(x^{\prime}\right)+\int_{-b}^{+b} f(x) \mathrm{K}\left(x, x^{\prime}\right) d x,
$$

où le noyau devient logarithmiquement infini quand $x$ tend vers $x^{\prime}$. Nous pouvons appliquer la théorie de Fredholm; elle dévoilera l'existence d'une solution continue de $(4,11)$, à condition que l'équation homogène associée n'ait pas de solutions extraordinaires. Pour nous rendre compte de ce qu'il n'y a point de telle solution, supposons qu'au contraire il $\mathrm{y}$ en a une et cherchons à en étudier les propriétés.

Soit $f_{o}\left(x^{\prime}\right)$ cette solution; formons la fonction :

$$
\psi_{o}\left(x^{\prime}, y^{\prime}\right)=\int_{-b}^{+b} f_{o}(x) \mathrm{G}\left(x, a, x^{\prime}, y^{\prime}\right) d x .
$$

De l'expression $(2,14)$ de $G$ conjointement avec le lemme (1), nous déduisons que $\psi_{0}\left(x^{\prime} y^{\prime}\right)$ est continu dans $0<y \leqslant a,-b<x^{\prime}<b$. De plus, $f_{0}(x)^{\prime}$ satisfait à $(4,11)$ quand son premier membre est posé égal à zéro.

Il s'en suit :

$$
\lim _{y^{\prime} \uparrow a} \frac{\partial \psi\left(x^{\prime}, y^{\prime}\right)}{\partial y^{\prime}}=0
$$

Mais :

$$
\frac{\partial \mathrm{G}\left(x, a, x^{\prime}, a\right)}{\partial y^{\prime}}-\mathrm{KG}\left(x, a, x^{\prime}, a\right)=0
$$

dans $-b<x<+b$, sauf en $x=x^{\prime}$ où la différence devient infinie et :

$$
\int_{-b}^{+b} f(x)\left[\frac{\partial \mathrm{G}\left(x, a, x^{\prime}, a\right)}{\partial y^{\prime}}-\mathrm{KG}\left(x, a, x^{\prime} a\right)\right] d x=f\left(x^{\prime}\right)
$$

Il en résulte :

$$
\lim _{y^{\prime} \uparrow a}\left\{\frac{\partial \psi_{0}\left(x^{\prime}, y^{\prime}\right)}{\partial y^{\prime}}-\mathbf{K} \psi\left(x^{\prime}, y^{\prime}\right)\right\}=f_{o}\left(x^{\prime}\right)
$$

(Il importe de noter que cette relation vaut aussi pour les fonctions $\psi$ et $f\left(x^{\prime}\right)$ qui résolvent notre problème; on peut donc déterminer directement $\psi\left(x^{\prime} a\right)$ quand on connaît $f\left(x^{\prime}\right)$, sans avoir à recourir à une nouvelle intégration.)

Nous allons enfin utiliser un lemme que nous énonȩons encore sans démonstration.

Lemme 2. - Soit $\varphi(x, y)$ une solution de $\varphi_{x x}+\varphi_{y y}-k^{2} \varphi=0$ pour $0<y<a$ satisfaisant aux conditions suivantes :

$$
\begin{aligned}
& \text { a) } \varphi y=0 \\
& \text { sur } \quad y=0 \\
& \text { b) } \varphi \underline{\eta}-\mathrm{K} \varphi=0 \\
& \text { sur } y=a,|x|>b \\
& \text { c) } \varphi y=0 \\
& \text { sur } \quad y=a,|x|<b \\
& \text { d) } \lim _{\mathrm{L} \rightarrow \infty}\left\{\int_{0}^{a}\left[\varphi_{x}(\mathrm{~L}, y)-i \mathrm{C} \varphi(\mathrm{L}, y)\right]^{2} d y+\int_{0}^{a}\left[\varphi_{x}(-\mathrm{L}, y)-i \mathrm{C} \varphi(-\mathrm{L}, y)\right]^{2} d y=0\right. \\
& \text { e) } \lim _{\varepsilon \rightarrow 0} \int_{\Gamma_{8}} \varphi \varphi_{n} d l=0
\end{aligned}
$$

où $\Gamma_{\varepsilon}$ se compose des hémisphères $(x \pm b)^{2}+y^{2}=\varepsilon, ! /<a$.

On a alors $\varphi(x, y) \equiv 0$ dans $0<y<a$.

Le lemme est a priori évident, puisqu'il indique simplement que s'il n'y a pas d'onde incidente et si la bande est maintenue rigide, il ne peut être créé de mouvement dans le fluide. On pourrait en trouver une preuve dans un lemme analogue donné par Fritz JoHN. 
La fonction $\psi_{o}\left(x^{\prime}, y^{\prime}\right)$ répond à l'hypothèse incluse dans ce lemme; elle est en fait continue en $( \pm b, a)$ donc identiquement nulle en $0<y^{\prime}<a$ et, par son caractère continu, aussi en $y=a$. Nous en déduisons $f_{o}\left(x^{\prime}\right) \equiv 0$ pour $-b<x^{\prime}<b$.

Il est curieux de noter que nous pouvons passer à la limite quand $k \rightarrow 0$ tout en conservant la même façon de procéder pour les grandes valeurs de $|x|$. Cela ne semble pas évident, puisque aux grandes distances les solutions de l'équation de Laplace se comportent tout à fait autrement que les solutions de $\varphi_{x p}+\varphi_{y y}-k^{2} \varphi=0$ quand $k>0$. Quand $k=0$ nous avons comme expression du potentiel $\varphi\left(x^{\prime}, y^{\prime}\right)$ :

$$
\varphi\left(x^{\prime}, y\right)=-\frac{1}{\pi} \int_{-b}^{+b} f(x)\left[\int_{0}^{\infty} \frac{\cosh w y^{\prime}}{\{\mathrm{K} \cosh \gamma a-\gamma \sinh \gamma a\}} \cos w\left|x-x^{\prime}\right| d w\right] d x
$$

où $f(x)$ satisfait ̀̀ :

$-i w_{o} \mathrm{~A} \cosh w_{0} a e^{i w_{o} x^{\prime}}=f\left(x^{\prime}\right)-\frac{\mathrm{K}}{\pi} \int_{-b}^{+b} f(x)\left[\int_{0}^{\infty} \frac{\cosh w a}{\mathrm{~K} \cosh w a-w \sinh w a} \cos w\left|x-x^{\prime}\right| d w\right] d x$

$w_{o}$ étant une solution de $\quad \mathrm{K} \cosh w_{o} a=w_{o} \sinh w_{o} a$.

Dans le cas d'une profondeur infinie, on peut évaluer le noyau explicitement et on obtient:

$$
\begin{array}{r}
-i w_{o} \mathrm{~A} e^{i w_{o} x^{\prime}}=f\left(x^{\prime}\right)-\frac{\mathrm{K}}{\pi} \int_{-b}^{+b} f(x)\left[\pi i e-i \mathrm{~K}\left|x-x^{\prime}\right|+\cos \mathrm{K}\left|x-x^{\prime}\right| \mathrm{Ci}\left(\mathrm{K}\left|x-x^{\prime}\right|\right)\right. \\
\left.+\sin \mathrm{K}\left|x-x^{\prime}\right|\left(\operatorname{Si}\left(\mathrm{K}\left|x-x^{\prime}\right|\right)-\pi / 2\right)\right] d x
\end{array}
$$

$\mathrm{Ci}$, Si étant les fonctions cosinus intégral et sinus intégral. Par son aspect le terme $\mathrm{Ci}\left(\mathrm{K}\left|x-x^{\prime}\right|\right)$ confirme le fait, noté précédemment, que le noyau devient infini logarithmique quand $x$ tend vers $x^{\prime}$.

Ayant ainsi établi l'existence d'une solution, on peut passer à la question de l'obtention des valeurs numériques. Une équation du type $(4,11)$ peut être résolue directement si l'on remplace $f(x)$ par un polynome approché; on obtient alors un système de $n$ équations linéaires permettant de déterminer $f(x)$ en $n$ points. La résolution numérique exige l'évaluation en chiffres d'intégrales comprenant $\mathrm{K}\left(x, x^{\prime}\right)$ et des puissances de $x$. La singularité ne gêne pas; on peut en effet l'exclure et traiter explicitement les intégrales qui la renferment. Des études de ce genre sont en cours actuellement et nous espérons pouvoir les faire connaître plus tard.

Un procédé plus élégant, bien que peut-être moins utile, consiste à utiliser les méthodes aux variations de Schwinger [7] qui ont été créés en vue de la résolution de problèmes de diffraction aux ouvertures. Si l'on multiplie l'équation $(4,11)$ par $f\left(x^{\prime}\right)$ et si on l'intègre le long de la bande, à l'aide de $(4,8)$, on trouve :

$\frac{1}{r}=\frac{w_{o}^{2} \sinh \gamma_{o} a}{\left(\mathrm{~K}+\gamma_{0} \mathrm{~J}^{\mathrm{b}} e^{-\gamma_{0} a}\right.}\left(1+\frac{2 \gamma_{0} a}{\sinh 2 \gamma_{o} a}\right) \frac{\mathrm{A}}{\mathrm{R}}=\frac{\int_{-b}^{+b} f\left(x^{\prime}\right)^{2} d x^{\prime}+\int_{-b}^{+b} \int_{-b}^{+b} f(x) f\left(x^{\prime}\right) \mathrm{K}\left(x, x^{\prime}\right) d x d x^{\prime}}{\left(\int_{-b}^{+b} f\left(x^{\prime}\right) e^{i w_{0} x^{\prime}} d x^{\prime}\right)^{2}}$

où $r$ est essentiellement le coefficient de réflexion. L'expression du second membre est insensible aux variations de premier ordre de $f(x)$ autour de sa valeur exacte, déduite de l'équation intégrale $(4,11)$. On peut donc espérer que le coefficient de réflexion sera peu sensible aux erreurs sur $f(x)$.

On peut exploiter de façon plus explicite le caractère stationnaire de $1 / r$. Développons $f(x)$ pour $(-b, b)$ en série de Fourier $\left(^{*}\right)$ :

$$
f(x)=\sum_{-\infty}^{+\infty} a_{n} e^{-i n x}
$$

$\left(^{\star}\right)$ Le choix de fonctions $/ e^{\star i n v}$ pour le développement de $f(x)$ n'est pas essentiel dans l'application de cette méthode, mais il s'impose puisque pour certains $w_{0}$ le groupe $\left\{B_{n}\right\}$ se réduit à un seul terme. 
Substituant cette expression dans $(4,11)$, on obtient :

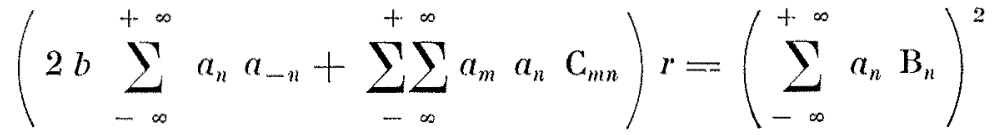

$$
\begin{aligned}
& \mathrm{C}_{m n}=\int_{-b}^{+b} \int_{-i n x}^{b} e^{-i m x^{\prime}} \mathrm{K}(x, x) d x d x, \quad \mathrm{~B}_{n}=\int_{-b}^{+b} e^{-i n x} e^{i w_{v} x} d x .
\end{aligned}
$$

Une différentiation par rapport à $a_{n}, r$ étant stationnaire, conduit à :

$$
\left(-2 a_{-m}+\sum_{-\infty}^{+\infty} a_{n} \mathrm{C}_{m n}\right) r=2 \mathrm{~B}_{m}\left(\sum_{-\infty}^{+\infty} a_{n} \mathrm{~B}_{n}\right) \quad m=0, \pm 1, \pm 2, \ldots
$$

Les constantes $\mathrm{D}_{p}$ étant définies par :

$$
a_{p}=\frac{2}{r} \mathrm{D}_{p}\left(\sum_{-\infty}^{+\infty} a_{n} \mathrm{~B}_{n}\right)=\frac{2 \mathrm{C}_{n}}{r} \mathrm{D}_{p} \quad p=0, \pm 1, \pm 2 \ldots
$$

on a finalement :

$$
\begin{aligned}
& r=2 \sum_{-\infty}^{+\infty} \mathrm{D}_{p} \mathrm{~B}_{p} \\
& \mathrm{D}_{-m}+\sum_{-\infty}^{+\infty} \mathrm{C}_{m n} \mathrm{D}_{n}=\mathrm{B}_{m} \quad m=0, \pm 1, \pm 2 \ldots
\end{aligned}
$$

Nous avons ainsi ramené le problème à la résolution d'un nombre infini d'équations linéaires telles que $(4,19)$. Les $\mathrm{D}_{n}$ ayant été trouvées, on pourra évaluer le coefficient de réflexion au moyen de $(4,18)$. Théoriquement on pourrait aussi obtenir $f(x)$. Si l'on introduit dans l'équation intégrale le développement en série de $f(x)$, avec $a_{p}$ remplacé par $\left(2 \mathrm{C}_{o} / r\right) \mathrm{D}_{p}$, on pourra déduire la constante $\mathrm{C}_{o}$ puis déterminer $a_{p}$.

Le procédé s'est révélé très efficace dans le cas de la diffraction des ondes sonores à travers une ouverture circulaire. Quelques termes de la série suffisent alors pour fournir une approximation suffisante.

\section{INDEX BIBLIOGRAPHIQUE}

1. John (F.). Communications on Applied Mathematics, vol. $3, \mathrm{n}^{\circ} 1,1950$.

2. Herns (A. E.). Canadian Journal of Mathematics, vol. 2, 1950.

3. Havelock (T.). Philosophical Magazine, vol. 8, 1929.

4. Kennard (E. H.). Quarterly of Appl. Math., vol. 7, $\mathrm{n}^{\circ} 3,1944$.

5. Wregel (R. L.). Gravity waves; tables of functions; Council of Waves Research, Berkeley, Calif., 1954.

6. Unsell (F.), Proceedings Cambridge Philosophical Society, t. 47, 1951.

7. Schwinger (J.). Physical Review, série 2, t. 74, oct. 1948. 


\title{
A source solution for short crested waves
}

\author{
BY R.C. MacCAMY \\ UNIVERSITY OF CALIFORNIA, WAVES RESEARCH LABORATORY.
}

Texte français, p. 367

\begin{abstract}
A Green's function for the boundary value problem arising in the diffraction of shortcrested waves around obstacles of bounded cross section is presented. The diffraction problem is formulated in a precise way which assures the existence and uniqueness of a solution. The Green's function is so constructed as to make possible a representation of the velocity potential at internal points of the fluid in terms of its values on the obstacle, thus in general reducing the diffraction problem
\end{abstract}

\author{
to the solution of Fredholm integral equation \\ of the second kind. Two problems of interest \\ in the theory of surface waves, the production \\ of waves by a moving partition, and the \\ reflection from a horizontal strip are studied \\ by means of the Green's function. Numerical \\ results are obtained for the first problem and \\ indications of numerical procedures given for \\ the second. In particular, the strip problem is \\ so formulated as to make possible the applic- \\ ation of the variational methods of Schwinger.
}

\section{I. - INTRODUCTION}

A difficult but fundamental problem in the theory of surface water waves is the study of diffraction around fixed obstacles. Such a situation arises in the theory of docks, piles, breakwaters and, as an auxiliary problem, in the study of ship motion. In three dimensional motion the problem has been attacked by Fritz JоHN [1] who succeeded in obtaining an integral equation formulation involving singular kernels. Beyond this little has been done for the case of obstacles of finite extent.

The purpose of the present report is to present a source solution in the case of the so-called short-crested waves, i. e., waves, which are periodic in a direction perpendicular to that of propagation. Such a solution is the fundamental tool in an investigation of associated diffraction problems by means of Fritz JoHs's methods. Physically, it gives the velocity potential of a line source on which the strength varies periodically along the line. Mathematically it enables one to express the potential of the interior of the fluid in terms of data on the obstacle surface.

In Section two we present the function and study its properties as well as indicate the general diffraction problems to which it is applicable. In the next two sections we indicate how the solution may be used in two problems, the determination of the waves produced by a wave-maker, and the reflection coefficient for a rigid horizontal barrier in the free surface. A few numerical results are given for the first problem.

The use of a source solution, or Green's function, is well known for other boundary value problems. In addition to yielding existence theorems which insure that the problem is correctly formulated, they may be used to obtain some approximate solutions. For example, it is shown in Section 4 that such a formulation enables one to invoke the variational procedures of Schwinger.

An effort has been made in this report to present ideas which admit of numerical computations. Some work of this kind is, in fact, underway now and will be presented at a later lime. 


\section{II. - THE GREEN'S FUNCTION}

In order to introduce the source solution in a natural way we will discuss briefly the general diffraction problem for short-crested waves in the presence of cylindrical obstacles.

Suppose an incompressible, non-viscous fluid fills the region:

$$
-\infty \leqslant x \leqslant+\infty,-\infty \leqslant Z \leqslant+\infty, \quad 0<y<a .
$$

The plane $y=0$ is to be a rigid bottom and $y=a$ a free surface. We assume the motion to be generated initially by a short-crested wave system, of small amplitude, progressing in a pos:tive $x$ direction. Such a system may be represented by by :

$$
\Phi^{(i)}=\mathrm{A} \cosh \gamma_{o} y e^{i\left(w_{o} x-\sigma t\right)} \begin{aligned}
& \cos \\
& \sin
\end{aligned} k \mathrm{Z}=\operatorname{Re}\left\{\varphi^{(i)} e^{-i \sigma}\right\}\left\{\begin{array}{l}
\cos \\
\sin
\end{array} k Z\right.
$$

where $\mathrm{A}$ is a constant and $\gamma_{o}, w_{o}$ are determined by :

$$
\mathrm{K}=\frac{\sigma^{2}}{g}=\gamma_{o} \tanh \gamma_{o} a, \quad w_{o}^{2}=\gamma_{o}^{2}-k^{2}
$$

It should be noted that Equation (2.2) puts an upper limit on $k$ in order that the waves progress in the $x$-direction, namely $k<\mathrm{K}$, and we shall henceforth assume this to be the case.

Now let a cylindrical obstacle of bounded cross section be fixed in the fluid. The resulting motion, once steady state is reached, will again be time periodic with frequency $\sigma$. Moreover, we reduce the problem to one in two-dimensions by assuming the $Z$ dependence to remain in the form $\cos k \mathrm{Z}$ or $\sin k \mathrm{Z}$. Under these assumptions we write for the velocity potential of the ensuing motion:

$$
\Phi(x, y, Z, t)=\operatorname{Re}\left(\varphi(x, y) e^{-i^{t} t}\right) \underset{\sin }{\cos k Z}
$$

and there results, for $\varphi$, the equation:

in fluid.

$$
\varphi_{x x}+\varphi_{y \prime \prime}-k^{2} \varphi=0
$$

If we denote by $\mathrm{C}_{o}$ the trace of the obstacle on the $x-y$ plane, and by $\mathrm{C}_{\mathrm{F}}$ that portion of $y=a$ exterior to $\mathrm{C}_{o}$, we find that $\varphi$ is also subject to the boundary conditions:

$$
\begin{array}{lll}
\varphi_{y}-\mathrm{K} \varphi=0 & \text { on } & \mathrm{C}_{\mathrm{F}} \\
\varphi_{y}=0 & \text { on } & y=0 \\
\varphi_{n}=0 & \text { on } & \mathrm{C}_{o}
\end{array}
$$

where $n$ denotes the normal to $\mathrm{C}_{0}$.

In order to fix the solution uniquely it is necessary to specify conditions at infinity. We would expect the motion to consist of an incident plus a reflected and transmitted wave, i.e., a condition of the form:

$$
\varphi-\varphi^{(i)} \rightarrow \mathrm{T} e^{i w_{0} x} \quad \text { as } x \rightarrow+\infty, \quad \varphi-\varphi^{(i)} \rightarrow \mathrm{Re}^{-i w_{0} x} \quad \text { as } x \rightarrow-\infty .
$$

It turns out to be sufficient to demand only the weaker condition:

$$
\lim _{\mathrm{L} \rightarrow \infty}\left\{\int_{0}^{a}\left[\psi_{x}(\mathrm{~L}, y)-i \mathrm{C} \psi(\mathrm{L}, y)\right]^{2} d y+\int_{0}^{a}\left[\psi_{x}(-\mathrm{L}, y)-i \mathrm{C} \psi(-\mathrm{L}, y)\right]^{2} d y\right\}=0
$$

for some $\mathrm{C}>0$, where $\psi=\varphi-\varphi^{(i)}$ represents the scattered wave. It can be shown that there is a 
unique $\varphi$ satisfying (2.4), (2.5), (2.6), (2.7) and (2.9) and that this solution does indeed satisfy (2.8).

The Green's function, $\mathrm{G}\left(x, y, x^{\prime} y^{\prime}\right)$, which depends on a parameter point $\left(x^{\prime}, y^{\prime}\right)$ is to be so constructed that it expresses values of solutions at $\left(x^{\prime}, y^{\prime}\right)$ in terms of data on the obstacle surface $\mathrm{C}_{0}$. It is to satisfy (2.5) on the entire $y=a$ plane, as well as (2.6), so as to suppress reference to values of the solution on $y=0$ and $\mathrm{C}_{\mathrm{F}}$. In addition it must have a singularity of a certain type for $(x, y)=\left(x^{\prime}, y^{\prime}\right)$ in order that the operation:

$$
\int_{-\infty}^{+\infty} \int_{0}^{a}\left(\mathrm{G}_{x x}+\mathrm{G}_{y y}-k^{2} \mathrm{G}\right) \varphi(x, y) d y d x
$$

reproduce solutions of (2.4). Finally G must carry the behavior of the solution for large $|x|$ and hence must satisfy (2.9).

The fundamental singularity of Equation (2.4) is found by separation of variables and is given by $K_{o}\left(k \sqrt{x^{2}+y^{2}}\right)$ where $K_{o}$ is essentially the Hankel function of order 0 and first type with pure imaginary argument. For our purpose, the important properties of $K_{o}(Z)$ are :

$$
\begin{gathered}
\mathrm{K}_{o}(Z)=0\left(e^{-z}\right) \text { for large } Z, \\
\mathrm{~K}_{o}(Z)=\mathrm{A}(Z) \log 1 / Z+\mathrm{B}(Z)
\end{gathered}
$$

where $A(Z), B(Z)$ are regular for real $Z$ and $A(0)=1$.

It can be shown that the Green's function as defined is unique and we proceed to give such a function. Consider:

$$
\begin{aligned}
& \mathrm{G}\left(x, y, x^{\prime}, y^{\prime}\right)=\frac{1}{\pi} \int_{0}^{\infty} \frac{\cosh \gamma y^{\prime}\{\mathrm{K} \sinh \gamma(a-y)-\gamma \cosh \gamma(a-y\}}{\gamma\{\mathrm{K} \cosh \gamma a-\gamma \sinh \gamma a\}} \cos w\left|x-x^{\prime}\right| d l b \\
& \text { for } y \geqslant y^{\prime} \\
& \frac{1}{\pi} \int_{0}^{\infty} \frac{\cosh \gamma y\left\{\mathrm{~K} \sinh \gamma\left(a-y^{\prime}\right)-\gamma \cos \gamma\left(a-y^{\prime}\right\}\right.}{\{\mathrm{K} \cosh \gamma a-\gamma \sinh \gamma a\}} \cos w\left|x-x^{\prime}\right| d w \\
& \text { for } y \leqslant y^{\prime}
\end{aligned}
$$

where $\gamma^{2}=w^{2}+k^{2}$ and $\mathrm{C}$ is a contour consisting of the positive $x$-axis except for a small semicircle around $w_{o}=\sqrt{\gamma_{o}^{2}-k^{2}}$ (which is real if $k<\mathrm{K}$ ). This function was given by Heins [2] in its partial fraction expansion form.

Making use of the identities:

$$
\begin{gathered}
\frac{\cosh \gamma \xi\{\mathrm{K} \sin \gamma(a-\zeta)-\gamma \cosh \gamma(a-\zeta)\}}{\gamma\{\mathrm{K} \cos \gamma a-\gamma \sinh \gamma a\}}=\frac{-(\mathrm{K}+\gamma) \cosh \gamma \xi \cosh \gamma \zeta e^{-\gamma a}}{\gamma\{\mathrm{K} \cos \gamma a-\gamma \sinh \gamma a\}} \\
\quad+\frac{e^{-\gamma(\zeta-\xi)}}{2 \gamma}+\frac{e^{-\gamma(\xi+\xi)}}{2 \gamma} \\
\int_{0}^{\infty} \frac{e^{--\sqrt{w^{2}+k^{2}} b}}{\sqrt{w^{2}+k^{2}}} \cos w x d w=\mathrm{K}_{o}\left(k \sqrt{\left.x^{2}+b^{*}\right)}\right.
\end{gathered}
$$

expression (2.12) may be transformed into:

$$
\begin{gathered}
\mathrm{G}\left(x, y, x^{\prime}, y^{\prime}\right)=\frac{1}{2 \pi} \mathrm{K}_{0}\left(k \sqrt{\left(x-x^{\prime}\right)^{2}+\left(y+y^{\prime}\right)^{2}}\right)+\frac{1}{2 \pi} \mathrm{K}_{0}\left(k \sqrt{\left(x-x^{\prime}\right)^{2}+\left(y+y^{\prime}\right)^{2}}\right) \\
-\frac{1}{\pi} \int_{0}^{\infty} \frac{(\mathrm{K}+\gamma) \cosh \gamma y \cosh \gamma y^{\prime} e^{-\gamma a}}{\gamma\{\mathrm{K} \cosh \gamma a-\gamma \sinh \gamma a\}} \cos w\left|x-x^{\prime}\right| d w
\end{gathered}
$$

We proceed to verify that. $G$ has the desired properties. From (2.14):

$$
G_{y}=0 \text { on } y=0 \text { and from (2.13) } \mathrm{G}_{y}-\mathrm{KG}=0 \text { on } y=a \text {, }
$$


Moreover, G satisfies (2.4) except at $\left(x^{\prime}, y^{\prime}\right)$ where it has a singularity of the prescribed type. In order to study the behavior of large $|x|$ it is convenient to make some further transformations on (2.14). We note that:

$$
\begin{aligned}
& \text { Res } \\
& w=w_{o}
\end{aligned}\left\{-\frac{1}{\pi} \frac{(\mathrm{K}+\gamma) \cosh \gamma u \cosh \gamma u^{\prime} e^{-\gamma u}}{\gamma(\mathrm{K} \cosh \gamma a-\gamma \sinh \gamma a)}\right\}=-\frac{1}{\pi} \frac{(\mathrm{K}+\gamma) e^{-\gamma_{o} u}}{w_{0} \sinh \gamma_{o} a} \frac{\cosh \gamma_{0} y \cosh \gamma_{n} u^{\prime}}{1+\frac{2 \gamma_{0} a}{\sinh 2 \gamma_{0} a}}
$$

thus we obtain, by shifting the contour, $\mathrm{C}$ :

$$
\begin{aligned}
& \mathrm{G}\left(x, y, x^{\prime}, y^{\prime}\right)=\frac{1}{2 \pi} \mathrm{K}_{o}\left(k \sqrt{\left(x-x^{\prime}\right)^{2}+\left(y-y^{\prime}\right)^{2}}\right)+\frac{1}{2 \pi} \mathrm{K}_{o}\left(k \sqrt{\left(x-x^{\prime}\right)^{2}+\left(y+y^{\prime}\right)^{2}}\right. \\
& -\frac{i(\mathrm{~K}+\gamma) e^{-\gamma_{o} a}}{w_{\mathrm{o}} \sinh \gamma_{o} a} \frac{\cosh \gamma_{o} y \cosh \gamma_{o} y^{\prime}}{1+\frac{2 \gamma_{0} a}{\sinh 2 \gamma_{0} a}} e^{i w_{o}\left|x-x^{\prime}\right|-\frac{1}{\pi}} \mathrm{Re} \int_{\mathrm{C}^{\prime}}^{\infty} \frac{(\mathrm{K}+\gamma) \cosh \gamma y \cosh \gamma y}{\gamma(\mathrm{K} \cosh \gamma a-\gamma \sinh \gamma a)} e^{i w_{o}\left|x-x^{\prime}\right| d w}
\end{aligned}
$$

where $\mathrm{C}^{\prime}$ is a contour lying in the first or fourth quadrant according as $x-x^{\prime}$ is $>0$ or $<0$. It follows, using (2.10) that,

$$
\mathrm{G} \sim-\frac{i\left(\mathrm{~K}+\gamma_{0}\right) e^{-\gamma_{0} a}}{w_{0} \sinh \gamma a} \frac{\cosh \gamma_{0} y \cosh \gamma_{0} y^{\prime}}{1+\frac{2 \gamma_{0} a}{\sinh 2 \gamma_{0} a}} e^{i \psi_{0} \mid x-v^{\prime}} \mid \text { as }|x| \rightarrow \infty
$$

Now the equation (2.2) has, in addition to the real roots $\pm \gamma_{0}$, an infinity of imaginary roots $\pm i \rho_{n}, n=1,2 \ldots$. . Consequently, although we make no use of the fact, the above transformation could be used to obtain an expansion of the integral term in a series of the functions $\left(^{*}\right)$.

In the case of infinite depth, the transformation may be carried somewhat further in order to yield an expression for $G$ involving only real integrals. For convenience, we change the coordinate system so that $y$ is measured vertically downward from the free surface. Then (2.15) becomes:

$$
\begin{aligned}
\mathrm{G}\left(x, y, x^{\prime}, y^{\prime}\right)=\mathrm{K}_{o}\left(k \sqrt{\left(x-x^{\prime}\right)^{2}+\left(y-y^{\prime}\right)^{2}}\right)-\frac{2 i \mathrm{~K}}{w w_{o}} e^{-\mathrm{K}\left(y+y^{\prime}\right)} e^{i w_{o}}\left|x-x^{\prime}\right| \\
--\frac{1}{\pi} \operatorname{Re} \int_{\mathrm{C}^{\prime}}^{\infty} \frac{(\gamma+\mathrm{K})}{\gamma(\mathrm{K}-\gamma)} e^{-\gamma\left(y+y^{\prime}\right)} e^{i w\left(x-x^{\prime}\right)} d w,
\end{aligned}
$$

where now $\mathrm{C}^{\prime}$ consists of the positive imaginary axis except for a small semi-circle in the right half plane about the singularity at $\gamma=0$, i.e., $w=i k$. Computing the real part one is led to,

$$
\begin{aligned}
& \mathrm{G}\left(x, y, x^{\prime}, y^{\prime}\right)=\frac{1}{2 \pi} \mathrm{K}_{o}\left(k \sqrt{\left(x-x^{\prime}\right)^{2}+\left(y-y^{\prime}\right)^{2}}\right)+\frac{1}{2 \pi} \mathrm{K}_{o}\left(k \sqrt{\left(x-x^{\prime}\right)^{2}+\left(y+y^{\prime}\right)^{2}}\right) \\
& +\frac{2 i \mathrm{~K}}{w_{o}} e^{-\mathrm{K}\left(y+y^{\prime}\right)} e^{i w\left(x-x^{\prime}\right)}-\frac{2 \mathrm{~K}}{\pi} \int_{l o}^{\infty}\left[\frac{\gamma \sin \gamma\left(y+y^{\prime}\right)+\mathrm{K} \cos \gamma\left(y+y^{\prime}\right)}{\gamma\left(\gamma^{2}+\mathrm{K}^{2}\right)} e^{-\tau\left(x-x^{\prime}\right)}\right] d \tau
\end{aligned}
$$

with $\gamma=\sqrt{\tau^{2}-k^{2}}$ and principal values of the integrals are meant, and where use has been made of (2.13).

\section{III. - WAVE GENERATION BY MOVING PARTITIONS}

As a first application we consider the generation of water waves in a basin by means of a vertical partition. It is assumed that the partition is divided into sections along its length, each of which may be moved with an independent amplitude and phase. If the sections are sufficiently

$\left(^{*}\right) \cos \rho^{n}\left(y-y^{\prime}\right) e^{\rho n \mid x-x^{\prime}}\left|, \cos \rho_{n}\left(y+y^{\prime}\right) e^{-\rho n}\right| x-x^{\prime} \mid$ 
small, the distribution of horizontal velocity of the partition may be given effectively an arbitrary shape. We suppose the entire partition to be sufficiently long that we may neglect end effects. The problem is to determine the motion, and the thrust needed to obtain that motion, which should be given the partition in order to produce a prescribed wave motion.

The solution is contained in the following result which is essentially a generalization of the work of Havelock [3] and Kennard [4].

Theorem. - The function $\varphi(x, y)$ defined by:

$$
\hat{\varphi}(x, y)=-2 \int_{0}^{\infty} f\left(y^{\prime}\right) \mathrm{G}\left(x, 0, x^{\prime}, y^{\prime}\right) d y^{\prime},
$$

is a solution of the problem:
a) $\varphi_{x w}+\varphi_{y j}-k^{2} \varphi=0$
b) $\varphi_{y}=0$
c) $\varphi_{y \prime}-\mathrm{K} \varphi=0$
d) $\varphi \rightarrow \mathrm{A} \cosh \gamma_{0} y e^{i w_{a, k}}$
e) $\lim _{x \rightarrow 0^{+}} \frac{\partial \varphi}{\partial x}=f(y)$

$$
\begin{array}{ll}
\text { in } & 0<x<\infty, 0<y<a \\
\text { on } & y=0, \quad x>0 \\
\text { on } & y=a, \quad x>0 \\
\text { as } & x \rightarrow \infty
\end{array}
$$

for $\quad 0<y<a$ and $f(y)$ continuous in $0<y<a$.

(2.16) with:

Proof. - The first three properties follow immediately from those of $G ;(d)$ follows from

$$
\mathrm{A}=+\frac{2 i(\mathrm{~K}+\gamma)}{w_{0} \sinh \gamma_{0} a} \frac{e^{-\gamma_{0} a}}{1+\frac{2 \gamma_{0} a}{\sinh 2 \gamma_{0} a}} \int_{0}^{a} f\left(y^{\prime}\right) \cosh \gamma_{0} y^{\prime} d y^{\prime}
$$

From (2.14) it follows that:

$$
\lim _{x \rightarrow 0^{+}} \frac{\partial \varphi}{\partial x}=\frac{1}{\pi} \lim _{x \rightarrow 0} \int_{0}^{a} f\left(y^{\prime}\right) \frac{\partial}{\partial x} \mathrm{~K}_{o}\left(k \sqrt{\left(x-x^{\prime}\right)^{2}+\left(y-y^{\prime}\right)^{2}}\right) d y^{\prime}
$$

On making use of (2.11) and the continuity of $f$ we can reduce this further to:

$$
\lim _{x \rightarrow 0^{+}} \frac{\partial 0}{\partial x}=\frac{1}{\pi} \lim _{x \rightarrow 0^{+}} f(y) \int_{-\infty}^{+\infty} \frac{x d x}{x^{2}+\left(y-y^{\prime}\right)^{2}}
$$

Then we make use of the fact that:

$$
\int_{-\infty}^{+\infty} \frac{x d x}{x^{2}+\left(y-y^{\prime}\right)^{2}}=\begin{array}{r}
\pi \text { for } x>0 \\
-\pi \text { for } x<0
\end{array}
$$

to obtain $\lim _{x \rightarrow 0^{+}} \frac{\partial \varphi}{\partial x}=f(y)$. This completes the proof and yields in addition the useful corollary:

Corollary. - If :

$$
\varphi(x, y)=-2 \int_{0}^{u} f\left(y^{\prime}\right) \mathrm{K}_{o}\left(k \sqrt{\left(x-x^{\prime}\right)^{2}+\left(y-y^{\prime}\right)^{2}}\right) d y^{\prime} \text { with } f(y)
$$

continuous:

$$
\lim _{x \rightarrow 0^{+}} \frac{\partial \varphi}{\partial x}=f(y), \quad \lim _{x \rightarrow 0^{+}} \frac{\partial \varphi}{\partial x}=-f(y)
$$

Formula (3.3) is a special case of a more general theorem which we will not develop here. Integrals of the above type represent "source layers" analogous to those occurring in ordinary potential theory, and formula (3.3) is merely a statement of the jump condition at such a layer. 
We are now in a position to consider the wave generation problem. To simplify the analysis we assume the generator is of the plunger type so that $f(y)=\mathrm{B}$, a constant. We denote by $v(y, z, t)$ the horizontal velocity of the partition and by $\eta_{\infty}$ the surface elevation at infinity.

Noting that :

$$
\eta=\left.\frac{1}{g} \frac{\partial \Phi}{\partial t}\right|_{y=0}
$$

our theorem enables us to consider several cases.

CASE (1). B real:

$$
\begin{aligned}
& v_{1}{ }^{c}=\mathrm{B} \cos \sigma t \cos k z, \quad \eta_{\infty}{ }^{1, c}=\frac{2 \sigma \mathrm{B} / g}{w_{0}\left[1+\left(2 \gamma_{0} a / \sinh \gamma_{0} a\right)\right]} \cos \left(w_{0} x-\sigma t\right) \cos k z \\
& v_{1}{ }^{s}=\mathrm{B} \cos \sigma t \sin k z, \quad \eta_{\infty}{ }^{1, *}=\frac{2 \sigma \mathrm{B} / g}{w_{0}\left[1+\left(2 \gamma_{0} a / \sinh \gamma_{0} a\right)\right]} \cos \left(w_{0} x-\sigma t\right) \sin k z
\end{aligned}
$$

CASE (2). B imaginary:

$$
\begin{aligned}
& v_{2}{ }^{c}=\mathrm{B} \sin \sigma t \cos k z, \quad \eta_{\infty}{ }^{2, c}=\frac{2 \sigma \mathrm{B} / g}{w_{0}\left\lceil 1+\left(2 \gamma_{0} a / \sinh \gamma_{0} \alpha\right)\right]} \sin \left(w_{0} x-\sigma t\right) \cos k z \\
& v_{2}{ }^{s}=\mathrm{B} \sin \sigma t \sin k z, \quad \gamma_{\infty}{ }^{2, s}=\frac{2 \sigma \mathrm{B} / g}{w_{0}\left\lceil 1+\left(2 \gamma_{0} a / \sinh \gamma_{0} a\right)\right\rceil} \sin \left(w_{0} x-\sigma t\right) \sin k z
\end{aligned}
$$

CASE (3). Superposition of $v_{1}{ }^{c}$ and $v_{2}{ }^{s}$ :

$$
\begin{aligned}
& v=v_{1}{ }^{\prime}+v_{2}{ }^{*}=\mathrm{B} \cos (k z-\sigma t) \\
& \eta_{\infty}=\eta_{\infty}^{1, s}+\eta^{2, s}=\frac{2 \sigma \mathrm{B} / g}{w_{0}\left\lceil 1+\left(2 \gamma_{0} a / \sinh \gamma_{0} a\right)\right\rceil} \cos \left(w_{0} x-k z-\sigma t\right)
\end{aligned}
$$

The first two cases represent situations in which the sections of the partition move with the same phase but varying amplitudes. The resulting wave motion, sufficiently far from the partition, will form a short-crested wave system propagating outward.

In the third case, on the other hand, the sections move with the same amplitude but differing phases. Te resulting system is now a plane wave moving outward at an angle, $\tan ^{-1} k / w$, to the $x$-axis, with frequency $\sigma$ and wave length $2 \pi / \gamma_{0}$.

If $\mu_{o}$ denotes the amplitude of the motion of the partition, we obtain from (3.5) and (3.6).

$$
\frac{\left|n_{\infty}\right|}{\mu_{o}}=\frac{2 \mathrm{~K}}{w_{0}} \frac{1}{1+\left(2 \gamma_{0} a / \sinh 2 \gamma_{0} a\right)}
$$

for the amplitude $\left|\eta_{\infty}\right|$, of the plane wave and in case (3). Now the items we are at liberty to specify are $\sigma$ and $k$, hence we take as parameters $K a=\left(\sigma^{2} a / 2 \pi g\right)$, and $k a$. For calculations the important parameters are $\mathrm{K} \alpha$ and $\left(k / w_{0}\right)=\beta$, the latter of which, as we have seen, measures the direction of propagation of the plane wave.

For water of infinite depth, $\gamma_{o}=\mathrm{K}$, and thus $\mathrm{K} a / 2 \pi$ is the ratio of the depth to deep water wave length of long-crested waves of frequency $\sigma$. This being the case, we can enter WiEgEL's [5] tables with $\mathrm{K} a / 2 \pi$ taking the place of $d / \mathrm{L}_{o}$.

A few results are indicated in Figure $1\left(^{*}\right)$.In Figure 2 the corresponding case of a flapper type generator, which is hinged at the bottom, is shown. It is noted that the wave-maker becomes increasingly less efficient as one attempts to increase the propagation angle. In fact, as $\theta$ approaches $45^{\circ}$ the ratio in (3.7) approaches 0 . If one attempts to produce greater angles one merely succeeds in generating so-called "edge waves" wich vanish exponentially away from the generator. A similar situation involving a submerged circular cylinder is considered in a recent paper by URSELL [6].

To obtain the pressure at the partition we can use:

(*) See French text, p. 373.

$$
p(\theta, y, z, t)=\left.\rho g \frac{\partial \Phi}{\partial t}\right|_{a=0}
$$


We obtain then:

$$
p=-\imath g \circ \operatorname{Re}\left\{i \mathrm{~B} \int_{0}^{a} \mathrm{G}\left(o, y, o, y^{\prime}\right) d y^{\prime} e^{-i a t}\right\} \begin{aligned}
& \cos \\
& \sin
\end{aligned} z
$$

an integral which could be obtained by quadrature.

\section{IV. - REFLECTION FROM A STRIP}

As a second application we consider the reflection of short crested waves from a strip of finite width rigidly fixed in the free surface. Mathematically the problem is the following:

$$
\begin{aligned}
& \varphi_{x: x}+\varphi_{y y}-k^{2} \varphi=0 \\
& \varphi_{y}=0 \\
& \varphi_{y}-\mathrm{K} \varphi=0 \\
& \varphi_{y}=0 \\
& \varphi-\mathrm{A} \cosh \gamma_{o} y e^{i w_{o x}}
\end{aligned}
$$

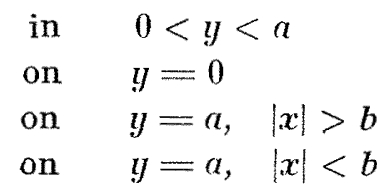

satisfies a condition of form $(2,9)$.

We remark at this stage that the analysis of Fritz John may be carried over almost intact to yield a fairly general existence theorem for the diffraction problem formulated in section two. This theorem, however, demands that the obstacle surface be perpendicular to the free surface at their intersection, a condition which is not satisfied in the present problem. Our construction will in itself constitute such an existence theorem for the present case.

Consider the function:

$$
\psi\left(x^{\prime}, y^{\prime}\right)=\int_{-b}^{+b} f(x) \mathrm{G}\left(x, a, x^{\prime}, y^{\prime}\right) d x+\mathrm{A} \cosh \gamma_{b} y^{\prime} e^{i w_{w} \cdot r}
$$

where $f(x)$ is a function we will determine later. From (2.16) we find,

$$
\begin{aligned}
& \psi\left(x^{\prime}, y^{\prime}\right) \sim \mathrm{A} \cosh \gamma_{o} y^{\prime} e^{i w_{o} x^{\prime}}-i \frac{\left(\mathrm{K}+\gamma_{n}\right) e^{-\gamma_{o} a}}{w_{o} \sinh \gamma_{o} a} \frac{\cosh \gamma_{0} y^{\prime}}{1+\left(2 \gamma_{0} a / \sinh 2 \gamma_{o} a\right)} e^{i w_{o} x^{*}} \int_{-b}^{+b} f(x) e^{-i w_{o} x} d x \\
& =\mathrm{A} \cosh \gamma_{0} y^{\prime} e^{i w_{0} x^{\prime}}+\mathrm{T}_{1} \cosh \gamma_{0} y^{\prime} e^{i w_{0} x^{\prime}} \quad \text { as } x \rightarrow+\infty \text {, }
\end{aligned}
$$

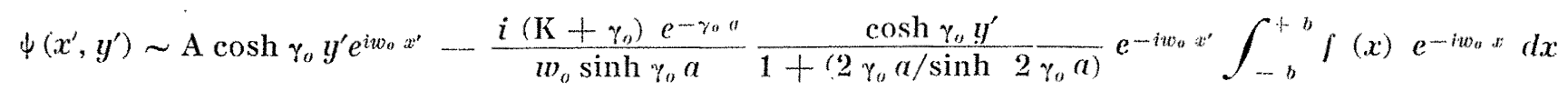

$$
\begin{aligned}
& =\mathrm{A} \cosh \gamma_{0} y^{\prime} e^{i w_{0} x^{\prime}}+\mathrm{R} \cosh \gamma_{0} y^{\prime} e^{-i w_{0} r^{\prime}} \quad \text { as } x \rightarrow-\infty \text {. }
\end{aligned}
$$

Thus $\psi\left(x^{\prime}, y^{\prime}\right)$ satisfies (4.5) with $\mathrm{R}$ representing a reflected wave and $\mathrm{T}_{1} \cosh \gamma_{0} y^{\prime} e^{i w w^{\prime}}$ combining with the incident wave to give a transmitted wave. That $\psi$ also satisfies (4.1) (4.2), and (4.3) may be verified by direct differentiation. In order to make $\psi\left(x^{\prime}, y^{\prime}\right)$ a solution of our problem it remains to show that $f(x)$ may be chosen in such a way that (4.4) is satisfied.

From the defining equation (2.12) for $G$ we find:

$$
\frac{\partial \mathrm{G}}{\partial y^{\prime}}\left(x, a, x^{\prime}, y^{\prime}\right)=-\frac{1}{\pi} \int_{0}^{\infty} \frac{\sinh \gamma y^{\prime}}{\mathrm{K} \cosh \gamma a-\gamma \sinh \gamma a} \cos w\left|x-x^{\prime}\right| d w .
$$

On differentiation of the identity (2.13) with respect to $b$ we find that (4.9) may be written as:

$$
\begin{aligned}
\frac{\partial \mathrm{G}}{\partial y^{\prime}}\left(x, a, x^{\prime}, y^{\prime}\right)= & +\frac{1}{\pi} \frac{\partial}{\partial y^{\prime}}\left\{\mathrm{K}_{o}\left(k \sqrt{\left(x-x^{\prime}\right)^{2}+\left(a-y^{\prime}\right)^{2}}\right)\right\} \\
& -\frac{1}{\pi} \int_{\sim}^{\infty}\left[\frac{\sinh \gamma y}{\mathrm{~K} \cosh \gamma a-\gamma \sinh \gamma a}+e^{-\gamma\left(a-y^{\prime}\right)}\right\rceil \cos w\left|x-x^{\prime}\right| d w .
\end{aligned}
$$



three.

We now state without proof the following result, part of wich was essentially proved in section

Lemma 1. - The function:

$$
\Phi\left(x^{\prime}, y^{\prime}\right)=\int_{-b}^{+b} f(x) \mathbf{K}_{o} \quad\left(k \sqrt{\left(x-x^{\prime}\right)^{2}+\left(a-y^{\prime}\right)^{2}}\right) d x
$$

for $f(x)$ continuous in $-b<x<+b$, is a solution of $\Phi_{a^{\prime} x^{\prime}}+\Phi_{y^{\prime} y^{\prime}}-k^{2} \Phi=0$ in $y^{\prime}<0$, is continuous on $y^{\prime}=a,-b<x<+b$ when defined as its limit value, from $y^{\prime}<a$ and satisfies:

$$
\lim _{\substack{y^{\prime} \rightarrow a \\ y^{\prime}<a}} \frac{\partial \Phi(x, y)}{\partial y^{\prime}}=\pi f(x) \text { for }-b<x^{\prime}<b .
$$

From this lemma and Equation (4.10) we have:

$$
\lim _{y^{\prime} \uparrow a} \frac{\partial \psi\left(x^{\prime}, y^{\prime}\right)}{\partial y^{\prime}}=f\left(x^{\prime}\right)+\int_{-b}^{+b} f(x) \mathrm{K}\left(x, x^{\prime}\right) d x+i w_{o} \mathrm{~A} \cosh \gamma_{o} a e^{i w_{o}} d
$$

where:

$\mathrm{K}\left(x, x^{\prime}\right)=-\frac{1}{\pi} \int_{0}^{\infty}\left[\frac{\gamma \sinh \gamma a}{\mathrm{~K} \cosh \gamma a-\gamma \sinh \gamma a}+1\right] \cos w\left|x-x^{\prime}\right| d w$

Setting

$$
=-\frac{\mathrm{K}}{\pi} \int_{0}^{\infty} \frac{\cosh \gamma a \cos w\left|x-x^{\prime}\right| d w}{\mathrm{~K} \cosh \gamma a-\gamma \sinh \gamma \alpha}
$$

$$
\lim _{y^{\prime} \uparrow^{a}} \frac{\partial \psi(x, w)}{\partial y^{\prime}}=0
$$

gives, therefore, an integral equation for the determination of $f(x)$.

Let us investigate the nature of the kernel, $\mathrm{K}\left(x, x^{\prime}\right)$. We write:

$$
\mathrm{K}\left(x, x^{\prime}\right)=-\frac{1}{\pi} \int_{\mathrm{C}^{\prime}}^{\infty} \frac{\cos w\left|x-x^{\prime}\right| d w}{1-(\gamma / \mathrm{K})} \frac{1}{\pi \mathrm{K}} \int_{\mathrm{C}^{\prime \prime}}^{\infty} \frac{\gamma \cos w\left(x-x^{\prime}\right)[\tanh \gamma a-1]}{[1-(\gamma / \mathrm{K}) \tanh \gamma a][1-(\gamma / \mathrm{K})]} d w
$$

where $C^{\prime}, C^{\prime \prime}$ consist of the positive real axis except for semi-circles in the lower half plane about the singularities. The first term then contains whatever difficulties may arise for $x=x^{\prime}$. Proceeding as in an earlier calculation, we find:

$$
\mathrm{K}\left(x, x^{\prime}\right)=\frac{1}{\pi} \int_{0}^{\infty} \frac{\cos w\left(x-x^{\prime}\right)}{\sqrt{w^{2}+k^{2}}} d w+\ldots-\ldots
$$

where the dots indicate terms which remain bounded as $x \rightarrow x^{\prime}$. Thus, according to Equation (2.13), $\mathrm{K}\left(x, x^{\prime}\right)$ behaves like $\mathrm{K}_{o}\left(k\left|x-x^{\prime}\right|\right)$ near $x^{\prime}=x$, and has a logarithmic singularity there.

We are thus confronted with the integral equation:

$$
-i w_{o} \mathrm{~A} \cosh \gamma_{o} a e^{i w_{o} x}=f\left(x^{\prime}\right)+\int_{-b}^{+b} f(x) \mathrm{K}\left(x, x^{\prime}\right) d x,
$$

in which the kernel becomes logarithmically infinite as $x \rightarrow x^{\prime}$. The Fredholm theory may be applied and will yield the existence of a continuous solution of (4.11) provided there exist no non-trivial solutions of the associated homogeneous equation. To see that no such solution exists, we suppose there were one and study its properties. Let $f_{o}\left(x^{\prime}\right)$ be the solution and form the function:

$$
\psi_{o}\left(x^{\prime}, y^{\prime}\right)=\int_{-b}^{+b} f_{o}(x) \mathrm{G}\left(x, a, x^{\prime}, y^{\prime}\right) d x
$$


From the expression (2.14) for G, and Lemma (1) we conclude that $\psi_{o}\left(x^{\prime}, y^{\prime}\right)$ is continuous in $0<y^{\prime} \leqslant a,-b<x^{\prime}<b$. Moreover $f_{o}\left(x^{\prime}\right)$ satisfies (4.11) with the left hand side set equal to 0 , hence:

$$
\lim _{y^{\prime} \uparrow a} \frac{\partial \psi\left(x^{\prime}, y^{\prime}\right)}{\partial y^{\prime}}=0
$$

Now:

$$
\frac{\partial \mathrm{G}\left(x, a, x^{\prime}, a\right)}{\partial y^{\prime}}-\mathrm{KG}\left(x, a, x^{\prime}, a\right)=0
$$

in $-b<x<+b$ except at $x=x^{\prime}$ where the difference becomes infinite in such a way that:

$$
\int_{-b}^{+b} f(x)\left[\frac{\partial \mathrm{G}\left(x, a, x^{\prime}, a\right)}{\partial y^{\prime}}-\mathrm{KG}\left(x, a, x^{\prime} a\right)\right] d x=f\left(x^{\prime}\right)
$$

Therefore:

$$
\lim _{y^{\prime} \uparrow a}\left\{\frac{\partial \psi_{0}\left(x^{\prime}, y^{\prime}\right)}{\partial y^{\prime}}-\mathrm{K} \psi\left(x^{\prime}, y^{\prime}\right)\right\}=f_{o}\left(x^{\prime}\right)
$$

(It is important to note that this relation holds also for the functions $\psi$ and $f\left(x^{\prime}\right)$ which are to solve our problem, a fact which makes possible a direct determination of $\psi\left(x^{\prime} a\right)$ once $f\left(x^{\prime}\right)$ is known, without recourse to another integration.)

Finally we make use of another lemma which we also state without proof: conditions:

Lemma 2: Let $\varphi(x, y)$ be a solution of $\varphi_{x \underline{x}}+\varphi_{y y}-k^{2} \xi=0$ in $0<y<a$ satisfying the following

a) $\varphi y=0$

b) $\varphi y-\mathrm{K} \varphi=0$

c) $\varphi y=0$

d) $\lim _{\mathrm{L} \rightarrow \infty}\left(\int_{0}^{a}\left[\varphi_{x}(\mathrm{~L}, y)-i \mathrm{C} \varphi(\mathrm{L}, y)\right]^{2} d y+\int_{0}^{a}\left[\varphi_{x}(-\mathrm{L}, y)-i \mathrm{C} \varphi(-\mathrm{L}, y)\right]^{2} d y=0\right.$

e) $\lim _{\varepsilon \rightarrow 0} \Gamma_{\Gamma_{\varepsilon}} \varphi \varphi_{n} d l=0$ where $\Gamma_{\varepsilon}$ consists of the hemispheres $(x \pm b)^{2}+y^{2}=\varepsilon, y<a$.

Then $\varphi(x, y) \equiv 0$ in $0<y<a$.

The lemma is intuitively obvious, since it merely states that if there is no incident wave and the strip is held rigid, no motion can be produced in the fluid. A proof may be derived from an analogous lemma given by Fritz John.

The function $\psi_{0}\left(x^{\prime}, y^{\prime}\right)$ enters the hypothesis of the lemma, in fact will be continuous at $( \pm b, a)$; thus it is identically 0 in $0<y^{\prime}<a$, and by the continuity also for $y^{\prime}=a$. We conclude $f_{o}\left(x^{\prime}\right) \equiv 0$ in $-b<x^{\prime}<b$.

It is interesting to note that we may pass to the limit as $k \rightarrow 0$, while still retaining the same type of behavior for large $|x|$. The fact does not seem obvious since the behavior of solutions of Laplace's equation at large distances is much different than that of solutions of $\varphi_{x x}+\varphi_{y y}-k^{2} \varphi=0$ for $k>0$. For $k=0$ we obtain for the potential $\varphi\left(x^{\prime}, y^{\prime}\right)$ :

$$
\varphi\left(x^{\prime}, y\right)=-\frac{1}{\pi} \int_{-b}^{+b} f(x)\left[\int_{0}^{\infty} \frac{\cosh w y^{\prime}}{\{\mathrm{K} \cosh \gamma a-\gamma \sinh \gamma a\}} \cos w\left|x-x^{\prime}\right| d w\right] d x
$$

where $f(x)$ satisfies :

$-i w_{o} \mathrm{~A} \cosh w_{o} a e^{i w_{o} x^{\prime}}=f\left(x^{\prime}\right)-\frac{\mathrm{K}}{\pi} \int_{-b}^{+b} f(x)\left[\int_{0}^{\infty} \frac{\cosh w a}{\mathrm{~K} \cosh w a-w \sinh w a} \cos w\left|x-x^{\prime}\right| d w\right] d x$

$w_{o}$ being a solution of

$\mathrm{K} \cosh w_{o} a=w_{o} \sinh w_{o} a$. 
If the depth is infinite the kernel may be evaluated explicitly and yields:

$$
\begin{array}{r}
-i w_{o} \mathrm{~A} e^{i w_{o} x^{\prime}}=f\left(x^{\prime}\right)-\frac{\mathrm{K}}{\pi} \int_{-b}^{+b} f(x)\left[\pi i e^{-i \mathrm{~K}}\left|x-x^{\prime}\right|+\cos \mathrm{K}\left|x-x^{\prime}\right| \mathrm{Ci}\left(\mathrm{K}\left|x-x^{\prime}\right|\right)\right. \\
\left.+\sin \mathrm{K}\left|x-x^{\prime}\right|\left(\operatorname{Si}\left(\mathrm{K}\left|x-x^{\prime}\right|\right)-\pi / 2\right)\right] d x
\end{array}
$$

where $\mathrm{Ci}, \mathrm{Si}$, are the integral-cosine and integral sine functions respectively. The appearance of the term $\mathrm{C} i\left(\mathrm{~K}\left|x-x^{\prime}\right|\right)$ confirms our earlier remark that the kernel becomes logarithmically infinite as $x \rightarrow x^{\prime}$.

The existence of a solution having been established, one may turn to the question of obtaining numerical results. An equation of the type (4.11) can be solved in a straightforward way by replacing $f(x)$ by a polynomial approximation and thus obtaining a system of $n$ linear equations for the determination of the value of $f(x)$ at $n$ points. The numerical work involves numerical computation of integrals involving $\mathrm{K}\left(x, x^{\prime}\right)$ and powers of $x$. The singularity gives no difficulty as it may be subtracted off and the integrals involving it carried out explicitly. Some work of this type is underway and it is hoped may be presented in the future.

A more elegant, although possibly less useful method is the application of the variational methods of ScHwinger [7], which were developed to treat aperture diffraction problems. If Equation (4.11) is multiplied by $f\left(x^{\prime}\right)$ and integrated over the strip and use is made of (4.8) we find,

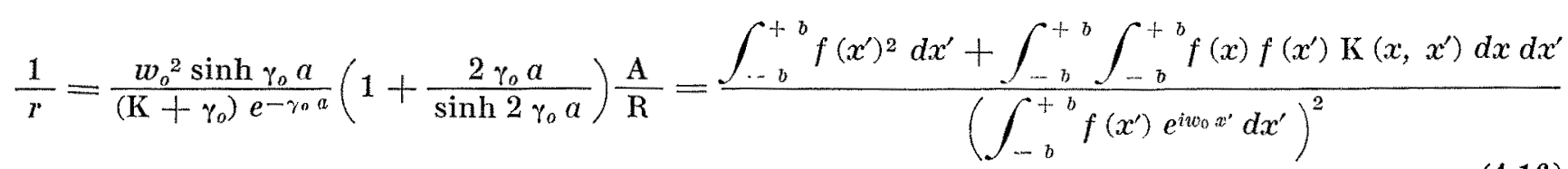

where $r$ is essentially the reflection coefficient. Now the quantity on the right hand side of (4.16) is stationary with respect to first order variations of $f(x)$ about its correct value as determined from the integral equation (4.11). It might be hoped, therefore, that the reflection coefficient would be relatively insensitive to errors in $f(x)$.

More explicit use of the stationary character of $1 / r$ may be made as follows. Suppose we expand $f(x)$ in $(-b, b)$ in a Fourier series $\left(^{*}\right)$ :

$$
f(x)=\sum_{-\infty}^{+\infty} a_{n} e^{-i n x}
$$

If we substitute this expression in (4.11) we obtain:

$$
\left(\begin{array}{llll}
2 b & \sum_{-\infty}^{+\infty} a_{n} & a_{-n}+\sum_{-\infty}^{+\infty} \sum_{m} a_{n} & a_{m n}
\end{array}\right) r=\left(\begin{array}{ccc}
\sum_{-\infty}^{+\infty} a_{n} & \mathrm{~B}_{n}
\end{array}\right)
$$

where:

$$
\mathrm{C}_{m n}=\int_{-b}^{+b} \int^{-i n x} e^{-i m x^{\prime}} \mathrm{K}(x, x) d x d x, \quad \mathrm{~B}_{n}=\int_{-b}^{+b} e^{-i n x} e^{i w_{0} \cdot x} d x .
$$

Differentiating with respect to $a_{n}$ and noting that $r$ is stationary:

$$
\left(-2 a_{-m}+\sum_{-\infty}^{+\infty} a_{n} \mathrm{C}_{m n}\right) r=2 \mathrm{~B}_{m}\left(\sum_{-\infty}^{+\infty} a_{n} \mathrm{~B}_{n}\right) \quad m=0, \pm 1, \pm 2, \ldots
$$

$\left.{ }^{\star}\right)$ The choice of functions $e^{ \pm \ln v}$ for the expansion of $f(x)$ is not essential to the method. It suggests itself, however, since for certain values of $w_{0}$, the set $\left\{B_{n}\right\}$ reduces to a single term. 
Defining constants, $\mathrm{D}_{p}$, by:

$$
a_{p}=\frac{2}{r} \mathrm{D}_{p}\left(\sum_{-\infty}^{+\infty} a_{n} \mathrm{~B}_{n}\right)=\frac{2 \mathrm{C}_{o}}{r} \mathrm{D}_{p} \quad p=0, \pm 1, \pm 2 \ldots
$$

we obtain finally:

$$
\begin{aligned}
& r=2 \sum_{-\infty}^{+\infty} \mathrm{D}_{p} \mathrm{~B}_{p} \\
& \mathrm{D}_{-m}+\sum_{-\infty}^{+\infty} \mathrm{C}_{m n} \mathrm{D}_{n}=\mathrm{B}_{m} \quad m=0, \pm 1, \pm 2 \ldots
\end{aligned}
$$

We have thus transformed the problem into solving an infinite system of linear equations, (4.19). Once the $\mathrm{D}_{n}$ 's are found the reflection coefficient can be computed from (4.18). In theory one could also obtain $f(x)$. Substituting the series for $f(x)$ with $a_{p}$ replaced by $\left(2 \mathrm{C}_{0} / r\right) \mathrm{D}_{p}$ in the integral equation fixes the constant $\mathrm{C}_{o}$ and thus determines $a_{p}$. For the case of diffraction of sound waves through a circular aperture, the scheme has proved quite successful, and it was found that only a few terms of the infinite series suffice to give quite accurate approximations.

\section{REFERENCES}

1. John (F.). Communications on Applied Math., vol. 3, $\mathrm{n}^{\circ} 1,1950$.

2. Heins (A. E.). Canadian Jour. of Math., vol. 2, 1950.

3. Havelock (T.). Philosophical Mag., vol. 8, 1929.

4. Kennard (E. H.). Quart. of Appl. Math., vol. 7, nº 3, 1949.

5. Wieger (R. L.). Gravity waves; tables of functions; Council on Wave Research, Berkeley, Cali1., 1954.

6. Ursell (F.). Proc. Cambridge Philos. Soc., vol. 47, 1951.

7. Schwinger (J.). Physical Review; series 2, vol. 74, Oct. 1948.

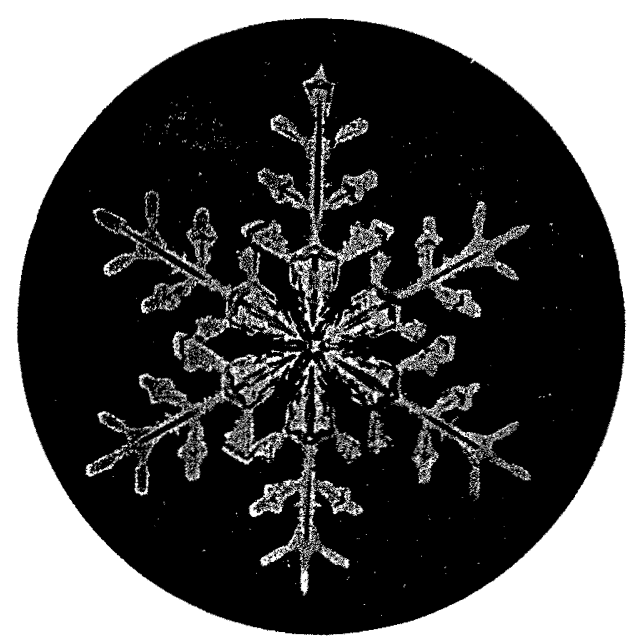

